\title{
A CLASS OF ALGEBRAS SIMILAR TO THE ENVELOPING ALGEBRA OF $\mathrm{sl}(2)$
}

\author{
S. P. SMITH
}

\begin{abstract}
Fix $f \in \mathbf{C}[X]$. Define $R=\mathbf{C}[A, B, H]$ subject to the relations

$$
H A-A H=A, \quad H B-B H=-B, \quad A B-B A=f(H) .
$$

We study these algebras (for different $f$ ) and in particular show how they are similar to (and different from) $U(\mathbf{s l}(2))$, the enveloping algebra of $\operatorname{sl}(2, \mathbf{C})$. There is a notion of highest weight modules and a category $\mathscr{O}$ for such $R$. For each $n>0$, if $f(x)=(x+1)^{n+1}-x^{n+1}$, then $R$ has precisely $n$ simple modules in each finite dimension, and every finite-dimensional $R$-module is semisimple.
\end{abstract}

\section{INTRODUCTION}

Fix $f \in \mathbf{C}[X]$. Define $R=\mathbf{C}[A, B, H]$ subject to the relations

$$
[H, A]=A, \quad[H, B]=-B, \quad A B-B A=f(H) .
$$

This paper studies these algebras (for different $f$ ) and in particular shows how they are similar to (and different from) $U(\operatorname{sl}(2))$, the enveloping algebra of $\mathbf{s l}(2, \mathbf{C})$. For example, each $R$ is a noetherian domain of Gelfand-Kirillov dimension 3 and has finite-dimensional simple modules of arbitrarily large finite dimension (whenever $f \notin \mathbf{C}$ ). Furthermore, there is a theory of Verma modules, highest weight modules, and a category $\mathscr{O}$ for $R$.

One reason for studying the rings $R$ is to construct examples of noetherian rings which have a rich structure that can be understood in detail and which exhibit some new features. Perhaps the most interesting part of the paper is $\S 5$, which analyzes the finite-dimensional $R$-modules. The results there show that the ideas involved in the study of enveloping algebras of semisimple Lie algebras have a wider applicability. One striking result is the following. Let $n>0$, and set $f(x)=(x+1)^{n+1}-x^{n+1}$. Then for each $d>0, R$ has precisely $n$ simple modules of dimension $d$, and every finite-dimensional $R$-module is semisimple (Example 5.10). The case $n=1$ gives $R \cong U(\operatorname{sl}(2))$. For general $R$ not every finite-dimensional module is semisimple. It is this feature which makes the structure of $R$ a little more interesting than that of $U(\operatorname{sl}(2))$.

Nevertheless, there are many similarities to $U(\mathbf{s l}(2))$, as is clear from the following brief description of the papers' contents.

Received by the editors November 27, 1988.

1980 Mathematics Subject Classification (1985 Revision). Primary 16A33, 16A05. 
$\S 1$. Description of $R$ as a skew polynomial ring over the enveloping algebra of the 2-dimensional nonabelian Lie algebra. $R$ is a noetherian domain of $G K$-dimension 3. The center of $R$ is a polynomial ring in one variable. $R$ is a subalgebra of the second Weyl algebra.

$\S 2$. Definition of highest weight modules, $V(\lambda)$, and the unique simple quotient of $V(\lambda), L(\lambda)$. Every finite-dimensional simple $R$-module occurs among the $L(\lambda)$. Description of which $L(\lambda)$ are finite-dimensional in terms of properties of $f$. The number of finite-dimensional simples of dimension $n$ is $\leq \operatorname{deg}(f)$. Central characters and homomorphisms between the $V(\lambda)$.

$\S 3$. The primitive ideals of $R$ are all of the form Ann $L(\lambda) ;\{\operatorname{Ann} V(\lambda)\}=$ \{minimal primitives\}, and Ann $V(\lambda)$ is generated by its intersection with the center. Each $R / \operatorname{Ann} V(\lambda)$ is completely prime and embeds in the first Weyl algebra with the same ring of fractions.

$\S 4$. There is a category of $R$-modules containing the $V(\lambda)$ and $L(\lambda)$ which is analogous to category $\mathscr{O}$ of Bernstein-Gelfand-Gelfand. The basic properties of category $\mathscr{O}$ hold in this context.

$\S 5$. A finite-dimensional $R$-module need not be semisimple. A nonsplit extension between two finite-dimensional simple modules must occur either at the "top" of some $V(\lambda)$ or at the "bottcm" of its dual. For certain $f$, every finite-dimensional $R$-module is semisimple.

$\S 6$. The case $\operatorname{deg}(f)=2$ is analyzed in detail.

$\S \S 1-3$ are mainly straightforward generalizations of the $U(\operatorname{sl}(2))$ theory, but are prerequisites for the main results of the paper, which occupy $\S \S 4-6$. Some useful identities (easy but tedious to verify) are given in an appendix.

This work was stimulated by a letter from Tim Hodges [H1] which followed on from the paper [AHV]. He was concerned with the action of the cyclic group $G=\mathbf{Z}_{m}$ as automorphisms of the Weyl algebra $\mathbf{C}[t, \partial=d / d t]$ (acting by $t \mapsto$ $\rho t, \partial \mapsto \rho^{-1} \partial$ where $\left.\rho=e^{2 \pi i / m}\right)$. The invariant subring is $\mathbf{C}\left[t^{m}, t \partial, \partial^{m}\right]$. As is well known, when $m=2$ this ring is isomorphic to a primitive factor of $U(\operatorname{sl}(2))$.

Hodges showed that there exist rings $S_{j}(1 \leq j \leq m)$ with $\mathbf{C}\left[t^{m}, t \partial, \partial^{m}\right] \subset$ $S_{j} \subset \operatorname{Frac} \mathbf{C}\left[t^{m}, t \partial, \partial^{m}\right]$ such that $\bigoplus_{j} S_{j}$ is a faithfully flat overring of $\mathbf{C}\left[t^{m}, t \partial, \partial^{m}\right]$. This is similar to the situation for (certain) primitive quotient rings $U(\mathfrak{g}) / P$ of the enveloping algebra of a semisimple Lie algebra $\mathfrak{g}$. The Beilinson-Bernstein equivalence of categories can be interpreted in terms of there being a certain faithfully flat overring of $U(\mathfrak{g}) / P$ [HS]. For example, this gives a faithfully flat overring of $\mathbf{C}\left[t^{2}, t \partial, \partial^{2}\right]$ consisting of a direct sum of two copies of the Weyl algebra $(\mathbf{C}[t, \partial]$ is not one of these); the two overrings correspond to the usual open affine cover of $\mathbf{P}^{1}$. Motivated by this analogy with $U(\mathfrak{g})$, I tried to find an algebra like $U(\mathbf{s l}(2))$ which would have the rings $\mathbf{C}\left[t^{m}, t \partial, \partial^{m}\right]$ as primitive quotients. Given $m$, there is indeed a suitable choice of $f$, such that $\mathbf{C}\left[t^{m}, t \partial, \partial^{m}\right]$ is a quotient of $R$ (Proposition 3.5). Since the first version of this paper was written, Hodges [H2] has shown that 
the primitive factors of all our rings $R$ possess such faithfully flat overrings, and has realized these primitive factors as the global sections of a sheaf of rings on a suitable (finite) topological space. Thus the primitive factor rings of $R$ can be studied locally in the same way as those of $U(\mathfrak{g})$.

Another reason for looking at these algebras is to construct new examples of noncommutative noetherian rings. One could begin by looking at some examples which are "small" in terms of various dimensions (Krull, GelfandKirillov, global homological); or one could look for examples which are "similar" to some known important examples (enveloping algebras, rings of differential operators); or one could look for examples which are given by generators and relations (few generators, and relations which on the surface look simple)-recent examples include the papers [AS, BS], and the examples in this paper fit into all three contexts. The value of the new examples can in part be judged in terms of whether they are tractable and exhibit interesting features. One useful test question is to classify the finite-dimensional simple modules (more generally the primitive ideals) and perhaps all finite-dimensional modules. This question is natural if one takes the following point of view. Suppose one wishes to find all matrices $X_{1}, \ldots, X_{n} \in M_{d}(\mathbf{C})$ which satisfy a given set of equations $f_{1}\left(X_{1}, \ldots, X_{n}\right)=\cdots=f_{r}\left(X_{1}, \ldots, X_{n}\right)=0$. This is the same as the problem of classifying all $d$-dimensional modules over the ring $\mathbf{C}\left\langle X_{1}, \ldots, X_{n}\right\rangle /\left(f_{1}, \ldots, f_{r}\right)$. Finally, as pointed out in (1.6), the rings $R$ which we construct can all be interpreted as rings of differential operators, so fit in with recent interest of ring theorists in differential operators.

\section{BASIC PROPERTIES OF $R$}

If $\operatorname{deg}(f) \leq 1$, then $R$ is familiar.

Proposition 1.1. If $\operatorname{deg}(f) \leq 1$, then $R$ is a factor ring of an enveloping algebra. In particular, if $A B-B A=\alpha H+\beta$ with $\alpha, \beta \in \mathbf{C}$, then

(a) if $\alpha=0$ and $\beta \neq 0$, then $R \cong \mathbf{C}[t, \partial] \otimes_{\mathbf{C}} \mathbf{C}[s]$ where $t$ and $s$ are commuting indeterminates, and $\partial=d / d t$;

(b) If $\alpha=0$ and $\beta=0$, then $R \cong U(\mathfrak{h})$ where $\mathfrak{h}$ is a 3-dimensional solvable Lie algebra;

(c) if $\alpha \neq 0$, then $R \cong U(\operatorname{sl}(2))$.

Proof. Define a Lie algebra $\mathfrak{g}$, with basis $A, B, H, X$ and relations

$$
[H, A]=A, \quad[H, B]=-B, \quad[A, B]=\alpha H+\beta X .
$$

There is a surjection $U(\mathfrak{g}) \rightarrow R$.

Notice that $X$ is a central element of $\mathfrak{g}$ and that $\mathfrak{g} / \mathbf{C} X$ is either solvable (when $\alpha=0$ ) or isomorphic to $\operatorname{sl}(2)$ (when $\alpha \neq 0$ ). Hence, if $\alpha=0, \mathfrak{g}$ is solvable, and if $\alpha \neq 0$, then $\mathfrak{g} \cong \mathrm{sl}(2) \oplus \mathbf{C}$. The rest is clear.

Henceforth we shall be interested in the case $\operatorname{deg}(f) \geq 2$.

Let $S$ be a ring and $\sigma \in$ Aut $S$. A $\sigma$-derivation of $S$ is a linear map $\delta: S \rightarrow S$ such that $\delta(s t)=s \delta(t)+\delta(s) t^{\sigma}$ for all $s, t \in S$. Given a $\sigma$-derivation, 
the skew polynomial ring determined by $\sigma$ and $\delta$ is the ring $S[X ; \sigma, \delta]:=$ $S\langle X\rangle /\left(s X-X s^{\sigma}-\delta(s) \mid s \in S\right)$. See [C, §12.2] for details. In particular, $S[X ; \sigma, \delta]=S \oplus S X \oplus S X^{2} \oplus S X^{3} \oplus \cdots$. The ring $S[X ; \sigma, \delta]$ is called a skew polynomial ring over $S$.

Proposition 1.2. Let $\mathfrak{b}$ be the 2-dimensional nonabelian Lie algebra, with basis $\{h, a\}$ and relation $[h, a]=a$. Then $R \cong U(\mathfrak{b})[X ; \sigma, \delta]$ is a skew polynomial ring over $U(\mathfrak{b})$, where $a^{\sigma}=a, h^{\sigma}=h-1, \delta(a)=f(h)$, and $\delta(h)=0$.

Proof. Observe that $\sigma$ extends to an automorphism of $U(\mathfrak{b})$ and that $\delta$ is a $\sigma$-derivation. The ring $U(\mathfrak{b})[X ; \sigma, \delta]$ is defined by the relations $h a-a h=a$, $a X=X a^{\sigma}+\delta(a)=X a+f(h)$, and $h X=X h^{\sigma}+\delta(h)=X h-X$. It is immediately clear that there is an isomorphism $R \rightarrow U(\mathfrak{b})[X ; \sigma, \delta]$ defined by $A \mapsto a, H \mapsto h, B \mapsto X$.

Set $\mathfrak{b}:=\mathbf{C} H+\mathbf{C} A$. This is a solvable Lie subalgebra of $R$ and will be the analog of a Borel subalgebra of $\mathrm{sl}(2)$.

Corollary 1.3. $R$ is a noetherian domain, with basis $\left\{A^{i} B^{j} H^{k} \mid(i, j, k) \in \mathbf{N}^{3}\right\}$, and $G K-\operatorname{dim} R=3$.

Proof. It is proved in [C] that if $S$ is a domain, so is $S[X ; \sigma, \delta]$, and if $S$ is noetherian, so is $S[X ; \sigma, \delta]$. Since $U(\mathfrak{b})$ is a noetherian domain so is $R$. Since $S[X ; \sigma, \delta]=S \oplus S X \oplus S X^{2} \oplus S X^{3} \oplus \cdots, R$ has a basis as claimed.

Let $\operatorname{deg}(f)=n$. Set $V=\mathbf{C}\left\langle A, 1, H, \ldots, H^{n}\right\rangle$, and $W=V \oplus \mathbf{C} B$.

Claim. $W^{k}=V^{k} \oplus V^{k-1} B \oplus V^{k-2} B^{2} \oplus \cdots \oplus V B^{k-1} \oplus \mathbf{C} B^{k}$. This is certainly true for $k=1$. The right-hand side is a direct sum and is contained in $W^{k}$. Since $B g(H)=g(H+1) B$ and $B A=A B+f(H) \in V B+V$, it follows that $B V \subset V B+V$. The claim now follows by induction by rewriting $W^{k}=$ $(V+\mathbf{C} B) W^{k-1}$.

Since $U(\mathfrak{b})$ is of $G K$-dimension 2, there is a polynomial, $p$ say, of degree 2 , such that $\operatorname{dim}_{\mathbf{C}} V^{k}=p(k)$. Now $\operatorname{dim}_{\mathbf{C}} W^{k}=p(0)+p(1)+\cdots+p(k)$ is certainly a polynomial of degree 3 .

J. T. Stafford pointed out that one could obtain the basis $A^{i} B^{j} H^{k}$ by Bergman [B, Theorem 1.2]. In the free algebra $\mathrm{C}\langle A, B, H\rangle$ assign degrees to the generators by $\operatorname{deg} A=\operatorname{deg} B=\operatorname{deg} f$ and $\operatorname{deg} H=1$. Order the monomials (the words in the letters $A, B, H$ ) first by degree and then lexicographically according to $A<B<H$. The relations defining $R$ give replacements $B A=A B-f(H), H B=B H-B, H A=A H+A$. There is only one ambiguity, namely $H B A$, and it is easy to check that this is resolvable. Hence a basis for $R$ is given by the irreducible monomials, namely the elements $A^{i} B^{j} H^{k}$.

The structure of $R$ depends on $f$, but it is useful to introduce another function of $H$, which we denote by $u$. Define a linear map $\Delta: \mathbf{C}[x] \rightarrow \mathbf{C}[x]$ by $(\Delta q)(x)=q(x+1)-q(x)$. Set $\left(\begin{array}{l}x \\ 0\end{array}\right)=1$, and for $n \geq 1,\left(\begin{array}{l}x \\ n\end{array}\right):=$ $x(x-1)(x-2) \cdots(x-n+1) / n$ ! The set $\left\{\left(\begin{array}{l}x \\ n\end{array}\right) \mid n \geq 0\right\}$ is a basis for $\mathbf{C}[x]$. Now $\Delta\left(\begin{array}{c}x \\ n\end{array}\right)=\left(\begin{array}{c}x \\ n-1\end{array}\right)$, so $\Delta$ is surjective, and $\operatorname{ker} \Delta=\mathbf{C}$. The next result is clear. 
Lemma 1.4. There exists $u \in \mathbf{C}[x]$ such that $f=\frac{1}{2} \Delta u$. Furthermore, for $k \in \mathbf{N}$, $u(x+1)-u(x+1-k)=f(x)+f(x+1)+\cdots+f(x-k+1)$.

Notation. For the rest of the paper $u$ will be as in (1.4), and $n=\operatorname{deg}(f)$ (thus $\operatorname{deg}(u)=n+1)$.

Remarks. (1) It will emerge that the behavior of finite-dimensional simple $R$ modules is determined by the behavior of the functions $u(x+1)-u(x+1-k)$ for $k \in \mathbf{N}$. For example, see (2.3), (2.4), (4.7).

(2) The operator $\Delta$ may be expressed as $\Delta=e^{D}-1$, where $D=d / d x$. Similarly, if $\Delta_{k}=e^{k D}-1$, then $\Delta_{k}: \mathbf{C}[x] \rightarrow \mathbf{C}[x]$ is surjective, and for $q \in$ $\mathbf{C}[x],\left(\Delta_{k} q\right)(x+1-k)=q(x+1)-q(x+1-k)$.

(3) The adjoint action $[H,-]$ of $H$ on $R$ gives a decomposition of $R$ into weight spaces $R=\bigoplus_{\nu \in \mathbf{Z}} R_{\nu}$ where $R_{\nu}:=\{r \in R \mid[H, r]=\nu r\}$. The commutant of $H$ is $R_{0}=\mathbf{C}[A B, B A, H]=\mathbf{C}[A B, H]=\mathbf{C}[A B+B A, H]$.

The center of $U(\mathbf{s l}(2))$ is a polynomial ring in 1-variable; there is an analogous result for $R$.

Proposition 1.5. Set $\Omega=A B+B A+\frac{1}{2}\{u(H+1)+u(H)\}$. The center of $R$ is $\mathrm{C}[\Omega]$.

Proof. It is straightforward to calculate that $\Omega$ is central. To see that the center equals $\mathbf{C}[\Omega]$, suppose that $z$ is central. Then

$$
z \in R_{0}=\mathbf{C}[A B+B A, H]=\mathbf{C}[\Omega, H] .
$$

Write $z=\sum_{i} \Omega^{i} c_{i}$ where $c_{i} \in \mathbf{C}[H]$. Then $0=[z, A]=\sum_{i} \Omega^{i}\left[c_{i}, A\right]=$ $A \sum_{i} \Omega^{i}\left\{c_{i}(H+1)-c_{i}(H)\right\}$. Hence, as $R$ is a domain, $c_{i}(H+1)-c_{i}(H)=0$ for all $i$. Thus each $c_{i}$ is a constant, and $z \in \mathbf{C}[\Omega]$.

If $M$ is an $R$-module on which $\Omega$ acts as scalar multiplication by $\alpha \in \mathbf{C}$, we say that $M$ has central character $\alpha$.

Proposition 1.6. There is an embedding $R \rightarrow \mathscr{D}\left(\mathbf{A}^{2}\right)=\mathbf{C}\left[x, y, \partial_{x}, \partial_{y}\right]$ given by $A \mapsto \frac{1}{2} y^{-1}\left(u(x+1)-u\left(x-y \partial_{y}+1\right)\right), H \mapsto x-y \partial_{y}$, and $B \mapsto y$. Thus, $R$ may be realized as an algebra of differential operators on the polynomial ring $\mathbf{C}[x, y]$.

Proof. Set $M:=\mathbf{C}[x, y]$ the polynomial ring in two variables. Define elements $a, b, h \in \operatorname{End}_{\mathbf{C}} M$ as follows:

$$
\begin{gathered}
a \cdot x^{i}=0, \quad \text { and for } j \geq 1, \\
a \cdot x^{i} y^{j}=x^{i}(f(x)+f(x-1)+\cdots+f(x-j+1)) y^{j-1} \\
=\frac{1}{2} x^{i}(u(x+1)-u(x-j+1)) y^{j-1} \\
b \cdot x^{i} y^{j}=x^{i} y^{j+1}, \quad h \cdot x^{i} y^{j}=x^{i}(x-j) y^{j} .
\end{gathered}
$$

These elements satisfy $h a-a h=a, h b-b h=-b, a b-b a-f(h)=0$. Hence, there is a ring homomorphism $R \rightarrow \operatorname{End}_{\mathrm{C}} M$. 
it is clear that $b=y$, and $h=x-y \partial_{y}$, and a little work shows that $a=\frac{1}{2} y^{-1}\left(u(x+1)-u\left(x-y \partial_{y}+1\right)\right)$. Hence there is a map $R \rightarrow \mathbf{C}\left[x, y, \partial_{y}\right]$. It remains to show this map is injective. Since $R$ is a noetherian domian with $G K-\operatorname{dim} R=3$, any proper factor ring of $R$ has $G K$-dimension at most 2 [KL, Proposition 3.5]. So it is enough to show that $G K-\operatorname{dim} \mathbf{C}[b a, b, h]=3$. Since $u\left(x-y \partial_{y}+1\right) \in \mathbf{C}[h]$ it is enough to show that $G K-\operatorname{dim} \mathbf{C}\left[u(x+1), y, y \partial_{y}\right]=3$. However, $\mathbf{C}\left[x, y, y \partial_{y}\right]$ is a finitely generated module over $\mathbf{C}\left[u(x+1), y, y \partial_{y}\right]$ and is of $G K$-dimension 3 , so it follows that $G K-\operatorname{dim} \mathbf{C}\left[u(x+1), y, y \partial_{y}\right]=$ 3 .

The homomorphism $R \rightarrow \mathscr{D}(\mathbf{C}[x, y])$ makes $\mathbf{C}[x, y]$ an $R$-module, which we call the universal Verma module.

In [MR] and [MS], a ring $R$ is said to be somewhat commutative if there is a filtration on $R$ by finite-dimensional subspaces such that the asssociated graded algebra is finitely generated and commutative. Since $R$ embeds in the second Weyl algebra, $R$ certainly has a filtration by finite-dimensional subspaces such that the associated graded algebra is commutative. It is not clear whether the associated graded algebra is finitely generated. However, given the description of $R$ in (1.2), it follows from [MR, Theorem 3.3] that $R$ is indeed somewhat commutative.

Proposition 1.7. $R$ is somewhat commutative.

The class of somewhat commutative algebras includes enveloping algebras and their factor rings, but it is considerably larger and includes many rings of differential operators. There is a good theory of Gelfand-Kirillov dimension, and a Hilbert-Samuel polynomial for the class of somewhat commutative algebras, and this is the point of [MS] and [MR]. In particular, there is a notion of "multiplicity" which behaves well on short exact sequences (see [MR] for details). We will make use of this in $\S 3$ to study the Krull dimension of factor rings of $R$.

\section{Highest WEIGHT MODULES AND FINITE-DIMENSIONAL SIMPLES}

Let $N$ be an $R$-module. For $\nu \in \mathbf{C}$, define the $\nu$-weight space of $N$ to be $N_{\nu}:=\{n \in N \mid H n=\nu n\}$. Call $N$ a highest weight module if there exists $\nu$ such that (a) $\operatorname{dim} N_{\nu}=1$, (b) $N=R N_{\nu}$, and (c) if $N_{\mu} \neq 0$, then $\nu-\mu \in \mathbf{N} \cup\{0\}$; the $\nu$ satisfying these conditions is unique and is called the highest weight of $N$. If $\nu-\mu \in \mathbf{N} \cup\{0\}$, then we will write $\nu \geq \mu$.

If $\lambda \in \mathbf{C}$, write $\mathbf{C}_{\lambda}$ for the 1-dimensional $\mathfrak{b}$-module killed by $H-\lambda$ and $A$. The Verma module of highest weight $\lambda$ is $V(\lambda):=R \otimes_{U(b)} \mathbf{C}_{\lambda}$. As a right $U(\mathfrak{b})$-module, $R$ is free on $1, B, B^{2}, \ldots$ Thus $V(\lambda) \cong \mathbf{C}[B]$ as a left $\mathrm{C}[B]$-module. The action of $H$ on $V(\lambda)$ is semisimple, and the highest weight space is $1 \otimes \mathbf{C}_{\lambda}$. Write $1 \otimes \mathbf{C}_{\lambda}=\mathbf{C} v_{\lambda}$. Each $B^{j} v_{\lambda}$ is of weight $\lambda-j$. Hence $V(\lambda)=\bigoplus_{j \in \mathrm{N} \cup\{0\}} V(\lambda)_{\lambda-j}$ is the sum of its weight spaces, $V(\lambda)$ is a highest 
weight module, and $\operatorname{dim}_{\mathbf{C}} V(\lambda)_{\lambda-j}=1$ for all $j \in \mathbf{N} \cup\{0\}$. The action of $A$ on a weight vector increases its weight by 1 .

Recall the universal Verma module $M=\mathbf{C}[x, y]$. For $\lambda \in \mathbf{C},(x-\lambda) M$ is an $R$-submodule of $M$. Thus $M /(x-\lambda) M$ is an $R$-module. It is easy to see that $M /(x-\lambda) M \cong V(\lambda)$.

The submodule structure of $V(\lambda)$ is described by

Lemma 2.1. Fix $\lambda \in \mathbf{C}$, and let $v_{\lambda}$ be a highest weight vector of $V(\lambda)$. The submodules of $V(\lambda)$ are precisely

$$
\left\{\mathbf{C}[B] B^{j} v_{\lambda} \mid u(\lambda+1)-u(\lambda-j+1)=0 \quad \text { for } j \in \mathbf{N}\right\} .
$$

Proof. Write $v=v_{\lambda}$. A submodule must be stable under the action of both $B$ and $H$. Because it is stable under $B$ it corresponds to an ideal of $\mathbf{C}[B]$. Because it is $H$-stable, it is the sum of its $H$-weight spaces. This forces the submodule to be of the form $\left(B^{j}\right) v$. This is a submodule precisely when it is stable under the action of $A$. This is equivalent to $A \cdot B^{j} v=0$. For $j \geq 1$,

$$
A \cdot B^{j} v=(B A+f(H)) B^{j-1} v=(B A+f(\lambda-j+1)) B^{j-1} v
$$

and by induction,

$$
A \cdot B^{j} v=(f(\lambda)+f(\lambda-1)+\cdots+f(\lambda-j+1)) B^{j-1} v .
$$

The lemma follows from the definition of the function $u$ in (1.4).

Corollary 2.2. (a) The length of $V(\lambda)$ is the number of distinct $j \in \mathbf{N} \cup\{0\}$ such that $u(\lambda+1)=u(\lambda-j+1)$. In particular, the length of $V(\lambda)$ is $\leq \operatorname{deg}(u)=$ $\operatorname{deg}(f)+1$.

(b) $V(\lambda)$ is a uniserial module. In particular, $V(\lambda)$ has a unique maximal submodule, and hence a unique simple quotient, which we denote $L(\lambda)$.

(c) $V(\lambda)$ has a simple socle.

Proof. (a) The submodules of $V(\lambda)$ are in 1-1 correspondence with those zeros of the polynomial $u(\lambda+1)-u(\lambda-x+1) \in \mathbf{C}[x]$ which are contained in $\mathbf{N} \cup\{0\}$. The degree of this polynomial is $\operatorname{deg}(u)$.

(b) This is immediate, because the only possible submodules are of the form $\left(B^{j}\right) v_{\lambda}$.

(c) This follows from (a) and (b).

Lemma 2.3. (a) The finite-dimensional simple $R$-modules are precisely the modules $L(\lambda)=V(\lambda) / B^{j} V(\lambda)$ where $j \in \mathbf{N}$ is minimal such that $u(\lambda+1)-$ $u(\lambda-j+1)=0$. (There may of course be no such $j$, in which case $L(\lambda)=V(\lambda)$.) If $\lambda \neq \nu$ then $L(\lambda) \not L(\nu)$.

(b) The number of simple modules of dimension $j$ equals

$\mid\{\lambda \in \mathbf{C} \mid u(\lambda+1)-u(\lambda-j+1)=0$, and $j$ is the least such element of $\mathbf{N}\} \mid$

which is $\leq \operatorname{deg}(u)-1=\operatorname{deg}(f)$. 
(c) If for each $j \in \mathbf{N}$ there are precisely $\operatorname{deg}(f)$ simple modules of dimension $j$, then, for all $\lambda \in \mathbf{C}$, length $V(\lambda) \leq 2$.

Proof. (a) Let $V$ be a finite-dimensional simple $R$-module. As a $U(\mathfrak{b})$-module, $V$ has a 1-dimensional submodule, $\mathbf{C} v$ say. By standard solvable Lie algebra theory, $A \cdot v=0$, and $H \cdot v=\lambda v$ for some $\lambda \in \mathbf{C}$. Since $V$ is generated by $v$, there is a surjection $V(\lambda) \rightarrow V$. Thus $V \cong L(\lambda)$.

If $\varphi: L(\lambda) \rightarrow L(\nu)$ is a nonzero module homomorphism then $\varphi\left(v_{\lambda}\right)$ must be annihilated by $(H-\lambda)$ and $A$. Hence $\varphi\left(v_{\lambda}\right)$ is a highest weight vector in $L(\nu)$ of weight $\lambda$. Thus $\lambda=\nu$.

(b) Obvious after (a).

(c) Fix $\lambda$. Suppose that length $V(\lambda) \geq 3$. Then there exist distinct $j_{1}, j_{2} \in \mathbf{N}$ such that $u(\lambda+1)-u\left(\lambda-j_{1}+1\right)=u(\lambda+1)-u\left(\lambda-j_{2}+1\right)=0$. Say $j_{1}<j_{2}$. Then $\lambda \notin\left\{\mu \in \mathbf{C} \mid u(\mu+1)-u\left(\mu-j_{2}+1\right)=0 \& j_{2}\right.$ is least such element of $\mathbf{N}\}$. Thus

$\mid\left\{\mu \in \mathbf{C} \mid u(\mu+1)-u\left(\mu-j_{2}+1\right)=0 \& j_{2}\right.$ is least such element of $\left.\mathbf{N}\right\} \mid \leq n-1$, and there are at most $n-1$ simple modules of dimension $j_{2}$. This is a contradiction.

Remarks. (1) Given $j \in \mathbf{N}$, there always exists $\lambda \in \mathbf{C}$ such that $u(\lambda+1)-$ $u(\lambda-j+1)=0$, so there is always an indecomposable $R$-module of dimension $j$. In particular, the 1-dimensional $R$-modules are $\{L(\lambda) \mid f(\lambda)=0\}$. However, there need not be a simple module of dimension $j$, as the next example illustrates.

(2) There exist simple $R$-modules of arbitrarily large finite dimension. To see this, suppose to the contrary that all finite-dimensional simple $R$-modules are of dimension $\leq k$. Fix $m \gg 0$. By (2.1) some $V(\lambda)$ has a quotient module, $M$ say, with $\operatorname{dim}_{\mathbf{C}} M=m$. By $(2.2 \mathrm{a}), \operatorname{deg}(f) \geq \operatorname{length}(M) \geq m / k$. The contradiction is clear.

Example 2.4. Let $u(x)=(x-1) x(x+1)$, so $f(x)=3 x(x+1) / 2$. Then $R$ does not have a simple module of dimension 2 .

Proof. Such a module would have to be a 2-dimensional quotient of $V(\lambda)$, where $\lambda$ satisfies $u(\lambda+1)-u(\lambda-1)=0$. Since $u(\lambda+1)-u(\lambda-1)=6 \lambda^{2}$, only $V(0)$ has a 2 -dimensional quotient. However, $V(0)$ has a 1-dimensional quotient, because $u(1)=u(0)$, and therefore (since $V(\lambda)$ is uniserial) the 2dimensional quotient of $V(0)$ is not simple.

In fact, $V(0)$ is the only Verma module of length 3 . Consequently, if $2 \neq$ $j \in \mathbf{N}$, there are exactly two simple modules of dimension $j$. To see this, set $\nu=\lambda+1$, and consider $u(\lambda+1)-u(\lambda-j+1)=j\left\{3 \lambda^{2}+3 \lambda(2-j)+(j-2)(j-1)\right\}=$ $j\left\{j^{2}-3 j \nu+3 \nu^{2}-1\right\}$. Thus $V(\lambda)$ has length $\geq 3 \Leftrightarrow$ there exist distinct $i, j \in \mathbf{N}$ which are zeroes of $y^{2}-3 y \nu+3 \nu^{2}-1$. This requires that $i+j=3 \nu$ and $i j=3 \nu^{2}-1$, and hence that $i^{2}-i j+j^{2}=3$. The only solution to this is $(i, j) \in\{(1,2),(2,1)\}$, and therefore $\nu=1$, so $\lambda=0$. Hence if $\lambda \neq 0$, 
then length $V(\lambda) \leq 2$. If $j \neq 2$, then there are two distinct $\nu$ such that $j^{2}-3 j \nu+3 \nu^{2}-1=0$.

Let $1<k \in \mathbf{N}$. I expect there exists $u$ with $\operatorname{deg}(u)=3$, such that $R$ has no simple module of dimension $k$.

I expect that for all except finitely many $j$, there are exactly $\operatorname{deg}(f)$ simple modules of dimension $j$. Unfortunately, I can only prove this is some special cases (see $\S 6$, and Example 5.10).

Proposition 2.5. The following are equivalent:

(a) there exists $k_{0} \in \mathbf{N}$ such that, if $j \in \mathbf{N}$, and $j>k_{0}$, then there are exactly $n$ simple modules of dimension $j$;

(b) there exists $k_{1} \in \mathbf{N}$ such that, if $j \in \mathbf{N}$, and $j>k_{1}$, then every highest weight module of dimension $j$ is simple;

(c) there exist only finitely many $\lambda \in \mathbf{C}$ such that length $M(\lambda)>2$.

Proof. Set $p(y, z)=u(y+1)-u(y+1-z)$. Then $M(\lambda)$ has a $j$-dimensional quotient $\Leftrightarrow p(\lambda, j)=0$, and the only $j$-dimensional highest weight modules are the $j$-dimensional quotients of those $M(\lambda)$ for which $p(\lambda, j)=0$. Let $D(z) \in \mathbf{C}[z]$ be the discriminant of $p(y, z)$ viewed as a polynomial in $y$, and set $Z=\{z \in \mathbf{C} \mid D(z)=0\}$. This set is finite, so choose $k \in \mathbf{N}$ such that if $j>k$, then $j \notin Z$. Hence, if $j \in \mathbf{N}$ and $j>k$, then there are precisely $n$ distinct highest weight modules of dimension $j$.

(a) $\Rightarrow(\mathrm{b})$ Let $j>k_{0}$. There are at most $n$ highest weight modules of dimension $j$. By hypothesis, each of these is simple, so take $k_{1}=k_{0}$.

(b) $\Rightarrow$ (c) Set $\mathscr{A}_{i}=\{\lambda \mid M(\lambda)$ has a quotient of dimension $i\}$. Then $\left|\mathscr{A}_{i}\right| \leq n$. Therefore $\mathscr{A}:=\mathscr{A}_{1} \cup \cdots \cup \mathscr{A}_{k_{1}}=\left\{\lambda \mid M(\lambda)\right.$ has a quotient of dimension $\left.\leq k_{1}\right\}$ is finite. Suppose $\lambda \notin \mathscr{A}$.

Claim. length $M(\lambda) \leq 2$. If $M(\lambda)$ is simple, this is true, so suppose that $M(\lambda)$ has a quotient of dimension $j$. Since $\lambda \notin \mathscr{A}, j>k_{1}$. Hence the quotient is simple, so length $M(\lambda) \leq 2$.

(c) $\Rightarrow$ (a) The set $\mathscr{A}=\{\lambda \mid$ length $M(\lambda) \geq 3\}$ is finite. Hence, so is the set

$$
K:=\bigcup_{\lambda \in \mathscr{A}}\{i \mid M(\lambda) \text { has a quotient of dimension } i\} .
$$

Set $k_{0}=\max \{i \in K, k\}$. Let $j>k_{0}$. Because $j>k$, there are $n$ distinct $\lambda_{1}, \ldots, \lambda_{n}$ such that each $M\left(\lambda_{q}\right)$ has a $j$-dimensional quotient. If some $\lambda_{q} \in \mathscr{A}$, then $j \in K$, a contradiction. Hence length $M\left(\lambda_{q}\right) \leq 2$, and the $j$-dimensional quotient of $M\left(\lambda_{q}\right)$ is simple.

Hence, the comment prior to the proposition amounts to an expectation that the equivalent conditions of Proposition 2.5 are satisfied for all $f$. To prove this one must show that a certain plane algebraic curve passes through only a finite number of integral points. The curve in question is

$$
\begin{aligned}
& V=\left\{(\alpha, \beta) \in \mathbf{C}^{2} \mid \alpha \neq \beta \text { and } \exists \lambda \in \mathbf{C}\right. \text { such that } \\
& \qquad \begin{array}{l}
u(\lambda+1)=u(\lambda+1-\alpha)=u(\lambda+1-\beta)\} .
\end{array}
\end{aligned}
$$


That is, I conjecture that $V \cap \mathbf{N}^{2}$ is finite for all $u \in \mathbf{C}[X]$ with $\operatorname{deg}(u) \geq 2$. This would follow from Siegel's theorem [L, p. 127, Theorem 4] if all the irreducible components of $V$ were of genus $\geq 1$, but this is not always the case.

Lemma 2.6. (a) The central character of $V(\lambda)$ is $u(\lambda+1)$.

(b) If $0 \rightarrow L(\lambda) \rightarrow X \rightarrow L(\nu) \rightarrow 0$ is a nonsplit extension, then $L(\lambda)$ and $L(\nu)$ have the same central character.

Proof. (a) Let $v_{\lambda} \in V(\lambda)$ be a highest weight vector. Set

$$
g(H)=\frac{1}{2}\{u(H+1)+u(H)\} .
$$

Then

$$
\begin{aligned}
\Omega \cdot v_{\lambda} & =(A B+B A+g(H)) \cdot v_{\lambda}=(A B+g(\lambda)) \cdot v_{\lambda} \\
& =(B A+f(\lambda)+g(\lambda)) \cdot v_{\lambda}=(f(\lambda)+g(\lambda)) v_{\lambda} .
\end{aligned}
$$

Hence, $\Omega$ acts on $V(\lambda)$ as multiplication by the scalar $f(\lambda)+g(\lambda)=u(\lambda+1)$.

(b) Suppose that $u(\lambda+1)=\alpha \neq \beta=u(\nu+1)$. Then $0 \neq(\Omega-\alpha) X \cong L(\nu)$, whence $X=L(\lambda) \oplus(\Omega-\alpha) X$ splits. Hence $\alpha=\beta$.

Lemma 2.7. If $\lambda, \nu \in \mathbf{C}$, then

(a) $\operatorname{dim}_{\mathrm{C}} \operatorname{Hom}_{R}(V(\nu), V(\lambda)) \leq 1$;

(b) $\operatorname{Hom}(V(\nu), V(\lambda))=\mathbf{C}$ if and only if $\nu=\lambda-j$ for some $j \in \mathbf{N}$, and $u(\lambda+1)-u(\lambda-j+1)=0$, i.e., $u(\lambda+1)=u(\nu+1)$.

(c) Every submodule of $V(\lambda)$ is of the form $V(\nu)$ for some $\nu$.

Proof. A homomorphism $\psi: V(\nu) \rightarrow V(\lambda)$ is completely determined by the image of the highest weight vector, $v_{\nu}$. If $\psi \neq 0$, then $\psi\left(v_{\nu}\right)$ is of weight $\nu$. Since $\operatorname{dim} V(\lambda)_{\nu} \leq 1, \operatorname{dim}_{\mathbf{C}} \operatorname{Hom}(V(\nu), V(\lambda)) \leq 1$. The dimension is 1 if and only if $V(\lambda)_{\nu} \neq 0$, and $A \cdot V(\lambda)_{\nu}=0$. Now $V(\lambda)_{\nu} \neq 0$ if and only if $\nu=\lambda-j$ for some $j \in \mathbf{N}$. In that case $V(\lambda)_{\nu}=B^{j} \cdot v_{\lambda}$. By (2.1), $A \cdot B^{j} v_{\lambda}=0$ if and only if $u(\lambda+1)-u(\lambda-j+1)=0$. This gives (a) and (b); (c) follows from (b) and the description of the submodules of $V(\lambda)$ given in (2.1).

Corollary 2.8. Let $\lambda, \nu \in \mathbf{C}$. Consider the following statements:

(a) $\operatorname{Hom}(V(\nu), V(\lambda))=\mathbf{C}$;

(b) there is an injective map $V(\nu) \rightarrow V(\lambda)$;

(c) $\nu=\lambda-j$ for some $j \in \mathbf{N} \cup\{0\}$, and $u(\lambda+1)=u(\nu+1)$;

(d) $V(\nu)$ and $V(\lambda)$ have the same central character;

(e) $u(\lambda+1)=u(\nu+1)$.

Then $(\mathrm{a}) \Leftrightarrow(\mathrm{b}) \Leftrightarrow(\mathrm{c}) \Rightarrow(\mathrm{d}) \Leftrightarrow(\mathrm{e})$.

\section{Primitive ideals of $R$}

Recall that if $\mathfrak{g}$ is a semisimple Lie algebra, and $0 \neq x \in U(\mathfrak{g})$; then there is a finite-dimensional simple $\mathfrak{g}$-module $E$ such that $x E \neq 0$. The next lemma is an analog of this for the ring $R$. 
Lemma 3.1. Let $\Lambda \subset \mathbf{C}$ be an infinite set with the property that $\operatorname{dim}_{\mathbf{C}} L(\lambda)<\infty$ for all $\lambda \in \Lambda$. Let $0 \neq x \in R$. Then $x L(\lambda) \neq 0$ for some $\lambda \in \Lambda$. Thus, if $\left\{I_{\lambda} \mid \lambda \in \Lambda\right\}$ is any infinite set of (distinct) ideals of finite codimension in $R$, then $\bigcap_{\lambda \in \Lambda} I_{\lambda}=0$.

Proof. Suppose that $x L(\lambda)=0$ for all $\lambda \in \Lambda$. Write $x=\sum_{\nu \in \mathbf{Z}} x_{\nu}$ where $x_{\nu}$ is a weight vector of weight $\nu$. If $v_{\mu} \in L(\lambda)$ is of weight $\mu$, then $x_{\nu} \cdot v_{\mu}$ is of weight $\nu+\mu$. Hence each $x_{\nu} \in \operatorname{Ann} L(\lambda)$. Fix $\nu$ with $x_{\nu} \neq 0$. By multiplying $x_{\nu}$ by a suitable power of $A$ or $B$, it follows that there is $0 \neq x \in R_{0}=$ $\mathrm{C}[H, \Omega]$ such that $x L(\lambda)=0$ for all $\lambda \in \Lambda$.

Fix $\lambda$, and write $\operatorname{dim}_{\mathbf{C}} L(\lambda)=d+1$. Then

$\mathbf{C}[H, \Omega] \cap \operatorname{Ann} L(\lambda)=\langle\Omega-u(\lambda+1),(H-\lambda)(H-\lambda-1) \cdots(H-\lambda-d)\rangle$.

There are only finitely many $L(\lambda)$ of a given dimension, hence $\left\{\operatorname{dim}_{\mathbf{C}} L(\lambda) \mid\right.$ $\lambda \in \Lambda\}$ is infinite. Similarly, given $\alpha \in \mathbf{C}$, there are only finitely many $\lambda$ with $u(\lambda+1)=\alpha$. Hence $\{\Omega-u(\lambda+1) \mid \lambda \in \Lambda\}$ is infinite. Therefore

$$
\bigcap_{\lambda \in \Lambda}\langle\Omega-u(\lambda+1),(H-\lambda)(H-\lambda-1) \cdots(H-\lambda-d)\rangle=0 .
$$

This contradicts the existence of $x$. Hence no such $x$ exists.

Notation. If $n \in \mathbf{N}$, write $\left(\begin{array}{c}H \\ n\end{array}\right)=(1 / n !) H(H-1) \cdots(H-n+1)$. The proof of (3.1) shows that if $\operatorname{dim} L(\lambda)=d+1$, then

$$
\mathbf{C}[H, \Omega] \cap \operatorname{Ann} L(\lambda)=\left\langle\Omega-u(\lambda+1),\left(\begin{array}{c}
H-\lambda+a^{\prime} \\
d+1
\end{array}\right)\right\rangle .
$$

A key point in the previous lemma is that any nonzero ideal of $R$ is stable under $[H,-]$ and hence contains a (nonzero) weight vector and therefore has nonzero intersection with $R_{0}=\mathbf{C}[H, \Omega]$. The following is a stronger version of this.

Theorem 3.2. If $0 \neq I$ is an ideal of $R$, then $I \cap \mathbf{C}[\Omega] \neq 0$.

Proof. As $R$ is noetherian, I contains a (finite) product of prime ideals, so it is enough to prove the result when $I$ is prime. Since $G K-\operatorname{dim} R=3$, it follows that $G K-\operatorname{dim}(R / I) \leq 2$.

Because $R$ is of countable dimension over $\mathbf{C}, I$ is the intersection of the primitive ideals which contain $I$. Furthermore, as the dimension of $R$ is countable, the center of any primitive factor ring of $R$ is $\mathbf{C}$, so if $I$ is primitive the result holds. Thus we may assume that $I$ is not primitive, and $I$ is the interesection of the primitive ideals which (strictly) contain $I$. If $P \supsetneqq I$ is primitive, then $G K-\operatorname{dim}(R / P) \leq 1$.

Sublemma. Let $S$ be a nonartinian, noetherian, finitely generated algebra over a field $k$, and supose that $G K-\operatorname{dim}(S)=1$. Then $S$ is not primitive.

Proof of Sublemma. Suppose that $S$ is primitive, and let $S / J$ be a faithful simple $S$-module. Since $\operatorname{dim}_{k} S=\infty$, so too is $\operatorname{dim}_{k}(S / J)=\infty$. If $J$ were not an essential left ideal, then the socle of $S$ would be nonzero, so would 
contain a regular element, $c$ say. But then $S \cong S c$ would be of finite length, contradicting the fact that $S$ is not artinian. Hence $J$ is essential, so contains a regular element $c$. But now $\operatorname{dim}_{k}(S / S c)=\infty$, and also $G K-\operatorname{dim}(S / S c) \leq$ $G K-\operatorname{dim} S-1$. This is a contradiction, because in this situation a module is of finite dimension if and only if it has $G K$-dimension zero.

Let $P \supsetneqq I$ be primitive. Thus $R / P$ is artinian, hence simple artinian. We wish to show that in fact $\operatorname{dim}_{\mathrm{C}}(R / P)<\infty$. Suppose not. Then there exists $a \in R / P$ which is a unit, and $a \notin \mathbf{C}$. Therefore the field $\mathbf{C}(a)$, of transcendence degree 1 , is contained in $R / P$. But $\mathbf{C}(a)$ is of uncountable dimension, contradicting the fact that $R$ is of countable dimension. Thus $\operatorname{dim}_{\mathbf{C}}(R / P)<\infty$. Hence $P$ is the annihilator of a finite-dimensional simple $R$-module. Thus $P=\operatorname{Ann} L(\lambda)$ for some $\lambda$.

Hence $I$ annihilates a collection of finite-dimensional $L(\lambda)$. But $I \neq 0$, so by the previous lemma, I can annihilate at most a finite number of such $L(\lambda)$. Thus there is only a finite number of primitive ideals $P \supsetneqq I$. But the intersection of a finite number of ideals of finite codimension in $R$ is again of finite codimension in $R$. Thus $\operatorname{dim}_{\mathbf{C}}(R / I)<\infty$, and certainly $I \cap \mathbf{C}[\Omega] \neq$ 0 .

Remark. We could have avoided the sublemma, and the paragraph after it, by an appeal to [SW]. The proof in [SW] works for a more general situation than that we are considering, and it seemed worthwhile to point out an easy proof of the following fact: Let $S$ be a finitely generated algebra of countable dimension over a field $k$. Suppose that $S$ is noetherian, primitive, and $G K-\operatorname{dim}(S) \leq 1$. Then $\operatorname{dim}_{k} S<\infty$, and $S$ is simple artinian.

Given the analogy with $U(\mathfrak{g})$, the next result is to be expected.

Theorem 3.3. (a) Every primitive ideal of $R$ is of the form Ann $L(\lambda)$.

(b) The minimal primitive ideals are the ideals Ann $V(\lambda)=(\Omega-u(\lambda+1))$.

(c) There is only a finite number of primitive ideals containing a given $(\Omega-$ $u(\lambda+1))$.

(d) If $I$ is an ideal such that $I \supsetneqq(\Omega-u(\lambda+1))$, then $\operatorname{dim}(R / I)<\infty$.

Proof. Recall the decomposition of $R$ into weight spaces: $R=\cdots \oplus B^{2} R_{0} \oplus$ $B R_{0} \oplus R_{0} \oplus A R_{0} \oplus A^{2} R_{0} \oplus \cdots$. As $V(\lambda)$ is the sum of its weight spaces, and $V(\lambda)$ is not annihilated by any power of either $A$ or $B$, it follows that Ann $V(\lambda)$ is generated by Ann $V(\lambda) \cap R_{0}$. But $\Omega-u(\lambda+1) \in$ Ann $V(\lambda)$, and $V(\lambda)$ has infinitely many distinct weights, so Ann $V(\lambda)=(\Omega-u(\lambda+1))$. Let $V(\nu)$ be isomorphic to the simple socle of $V(\lambda)$. Then $u(\nu+1)=u(\lambda+1)$, so Ann $V(\nu)=(\Omega-u(\nu+1))=(\Omega-u(\lambda+1))=$ Ann $V(\lambda)$. Hence Ann $V(\lambda)$ is a primitive ideal. Given any $\alpha \in \mathbf{C}$, there exists $\lambda \in \mathbf{C}$ such that $u(\lambda+1)=\alpha$; thus $(\Omega-\alpha)$ is a primitive ideal.

Let $P$ be a primitive ideal of $R$. Then $P \neq 0$, because the center of a primitive factor ring of $R$ must be $C$. Thus $P \cap C[\Omega] \neq 0$ and so contains $\Omega-\alpha$ for some $\alpha \in \mathbf{C}$. Hence $P \supset(\Omega-\alpha)$ for some $\alpha \in \mathbf{C}$. Thus the ideals $(\Omega-\alpha)$ are precisely the minimal primitive ideals of $R$. 
Fix $\alpha \in \mathbf{C}$. Let $P \supsetneqq(\Omega-\alpha)$ be a primitive ideal. By the argument in the proof of (3.2), $P=\operatorname{Ann} L(\lambda)$ for some finite-dimensional $L(\lambda)$. Thus every primitive ideal is of the form Ann $L(\lambda)$. Furthermore, since $u(\lambda+1)=\alpha$, there are only finitely many $P$ containing a given $\Omega-\alpha$.

Let $I \supsetneqq(\Omega-\alpha)$ be an ideal. Since $R$ is noetherian, $I$ contains a product of prime ideals, each of which contains $I$. Hence, to prove (d), we may suppose that $I$ is prime. But in $R$, every prime contains an intersection of primitive ideals. As the primitive ideals containing $I$ are all of finite codimension in $R$ and there are only finitely many of them, the result follows. In fact, it also follows that if $I$ is prime then $I$ is primitive.

For each $\lambda \in \mathbf{C}$, write $J(\lambda):=\operatorname{Ann} L(\lambda)$.

Lemma 3.4. Each $J(\lambda)$ is generated, as an ideal, by $J(\lambda)_{0}:=J(\lambda) \cap R_{0}$. Proof. If $\operatorname{dim}(R / J(\lambda))=\infty$, then this follows from (3.3b). So suppose that $\operatorname{dim}_{\mathrm{C}} L(\lambda)=d+1$.

Set $J(\lambda)_{i}=J(\lambda) \cap R_{i}$. Every ideal of $R$ is the sum of its ad $H$-weight spaces, so $J(\lambda)=\bigoplus_{i} J(\lambda)_{i}$. Recall that $R_{0}=\mathbf{C}[\Omega, H]$ and for $i \geq 0, R_{i}=$ $\mathrm{C}[\Omega, H] A^{i}$. and $R_{-i}=\mathbf{C}[H, \Omega] B^{i}$. By (3.1),

$$
J(\lambda)_{0}=\left\langle\Omega-u(\lambda+1)\left(\begin{array}{c}
H-\lambda+d \\
d+1
\end{array}\right)\right\rangle .
$$

More generally, for $d \geq i \geq 0$,

$$
J(\lambda)_{i}=\left\langle\Omega-u(\lambda+1),\left(\begin{array}{c}
H-\lambda+d-i \\
d-i+1
\end{array}\right)\right\rangle A^{i}
$$

and

$$
J(\lambda)_{-i}=\left\langle\Omega-u(\lambda+1),\left(\begin{array}{c}
H-\lambda+d \\
d-i+1
\end{array}\right)\right\rangle B^{i} .
$$

If $i>d+1$, then $J(\lambda)_{i}=R_{i}$, and $J(\lambda)_{-i}=R_{-i}$.

Now $R J(\lambda)_{0} R$ contains

$$
(\operatorname{ad} A)^{i}\left(\begin{array}{c}
H-\lambda+d \\
d+1
\end{array}\right)=(-1)^{i}\left(\begin{array}{c}
H-\lambda+d-i \\
d-i+1
\end{array}\right) A^{i} .
$$

The rest of the argument is clear.

Proposition 3.5. Let $u(x)=2(-1)^{m+1} m^{m}(x-1)(x-1+1 / m) \cdots(x-1 / m)$. Then there is a surjective map $R \rightarrow \mathbf{C}\left[t^{m}, t \partial, \partial^{m}\right]$ with kernel $\langle\Omega\rangle$.

Proof. Observe that $\left[-(1 / m) t \partial, t^{m}\right]=-t^{m},\left[-(1 / m) t \partial, \partial^{m}\right]=\partial^{m}$ and, setting $h=-(1 / m) t \partial,\left[\partial^{m}, t^{m}\right]=\frac{1}{2}\{u(h+1)-u(h)\}$. Hence there is a surjection as claimed; the map is $A \mapsto \partial^{m}, B \mapsto t^{m}, H \mapsto h$. The natural action of $\mathbf{C}\left[t^{m}, t \partial, \partial^{m}\right]$ on $\mathbf{C}\left[t^{m}\right]$ is faithful, and $\mathbf{C}\left[t^{m}\right] \cong V(0)$, which has central character $u(1)=0$.

The fact that this primitive factor embeds in the Weyl algebra $\mathbf{C}[t, \partial]$ is typical of what happens in general. Recall the definition of the map $R \rightarrow$ $\mathscr{D}(\mathbf{C}[x, y])$ in (1.6). The image is contained in $\mathbf{C}\left[x, y, \partial_{y}\right]$. For each $\lambda \in \mathbf{C}$, 
there is a 2 -sided ideal $\langle x-\lambda\rangle$ in this ring and $\mathbf{C}\left[x, y, \partial_{y}\right] /\langle x-\lambda\rangle \cong \mathbf{C}[y, \partial]$ where $\partial=d / d y$. Thus, composing, there is a homomorphism $R \rightarrow \mathbf{C}[y, \partial]$. As remarked earlier, the natural action of $\mathbf{C}\left[x, y, \partial_{y}\right]$ on $M=\mathbf{C}[x, y]$ then induces an action of $R /\langle x-\lambda\rangle \cap R$ on $M /\langle x-\lambda\rangle M=\mathrm{C}[y] \cong V(\lambda)$. Since $\mathbf{C}[y]$ is a faithful $\mathbf{C}[y, \partial]$-module, it is a faithful $R /\langle x-\lambda\rangle \cap R$ module. Thus $\langle x-\lambda\rangle \cap R \subset$ Ann $V(\lambda)$. Consider the action of $\Omega$ on $\mathbf{C}[x, y]$. Noting that $g(H) \cdot x^{i} y^{j}=g(x-j) x^{i} y^{i}$, it follows that $\langle\Omega-u(x+1)\rangle \cdot x^{i} y^{j}=0$. But the annihilator of $\mathbf{C}[x, y]$ in $\mathbf{C}\left[x, y, \partial_{y}\right]$ is $\langle x-\lambda\rangle$. Hence $\Omega-u(x+1) \in\langle x-\lambda\rangle$, and thus

$$
\Omega-u(\lambda+1) \in\langle x-\lambda\rangle \cap R \subset \operatorname{Ann} V(\lambda)=\langle\Omega-u(\lambda+1)\rangle .
$$

Hence, $\langle\Omega-u(\lambda+1)\rangle=\langle x-\lambda\rangle \cap R=\operatorname{Ann} V(\lambda)$.

The fact that these primitive factors embed in the Weyl algebra is similar to what happens for enveloping algebras. There has recently been some interest in realizing more of the primitive factor rings of $U(\mathfrak{g})$ as rings of differential operators (see [J, LSS, LS]).

Proposition 3.6. Set $\alpha=u(\lambda+1)$. There is an injection $R /\langle\Omega-\alpha\rangle \rightarrow \mathbf{C}[y, \partial]$ given by $B \mapsto y, H \mapsto \lambda-y \partial$, and $A \mapsto y^{-1}(\alpha-u(\lambda-y \partial+1))$. As an R-module $\mathrm{C}[y] \cong V(\lambda)$. Furthermore, $R /\langle\Omega-\alpha\rangle$ is a domain, and Fract $R /\langle\Omega-\alpha\rangle \cong$ Fract $\mathbf{C}[y, \partial]$.

Proof. This follows from (1.6). That the rings of fractions are the same follows from the fact that $\mathbf{C}[y, y \partial] \subset R /\langle\Omega-\alpha\rangle \subset \mathbf{C}[y, \partial]$.

The filtration on $\mathbf{C}[y, \partial]$ induces a filtration on $R /\langle\Omega-\alpha\rangle$. Write $\mathbf{C}[y, \xi]$ for the associated graded ring of $\mathbf{C}[y, \partial]$. Then

$$
\operatorname{gr} R /\langle\Omega-\alpha\rangle=\mathbf{C}\left[y, y \xi, y^{n} \xi^{n+1}\right]
$$

where $n=\operatorname{deg}(f)$. There are two alternative descriptions worth noting:

(a) $\operatorname{gr} R /\langle\Omega-\alpha\rangle \cong \mathbf{C}[X, Y, Z] /\left\langle X Y-Z^{n+1}\right\rangle$;

(b) $\operatorname{gr} R /\langle\Omega-\alpha\rangle \cong \mathbf{C}\left[X^{n}, Y^{n}, X Y\right] \subset \mathbf{C}[X, Y]$.

Again, the analogy with $U(\operatorname{sl}(2))$ is clear.

For certain $\alpha \in \mathbf{C}$, there are curves $X$ (some singular) and isomorphisms $U(\operatorname{sl}(2)) /\langle\Omega-\alpha\rangle \cong \mathscr{D}(X)^{\mathbf{Z}_{2}}$, the invariant subalgebra for a suitable action of $\mathbf{Z}_{2}$ as automorphisms of the ring $\mathscr{D}(X)$ of differential operators on $X$ (see [Sm] for details). I expect there are similar results for the appropriate primitive quotients of the rings $R$.

If $\mathfrak{g}$ is a semisimple Lie algebra, and $P$ a primitive ideal of $U(\mathfrak{g})$, then $U(\mathfrak{g}) / P$ contains a unique minimal nonzero ideal.

Theorem 3.7. Let $\alpha \in \mathbf{C}$, and set $S=R /\langle\Omega-\alpha\rangle$. Then $S$ has a unique minimal nonzero ideal.

Proof. We will show that there exists $0 \neq r \in \mathbf{C}[H]$ such that, if $0 \neq I$ is an ideal of $S$, then $r \in I$. Hence the intersection of all the nonzero ideals of $S$ is nonzero as required. 
Let $I \neq 0$ be an ideal of $S$. By applying ad $H$ to an element of $I$, it follows that $I$ contains a nonzero weight vector. By multiplying this weight vector by a suitable power of $A$ or $B$, it follows that $I$ contains a nonzero element of weight 0 . Since $R_{0}=\mathrm{C}[H, \Omega]$ it follows that $\mathbf{C}[H]$ is the weight 0 part of $S$. Hence there exists $0 \neq g(H) \in I \cap \mathbf{C}[H]$. Thus $I$ contains $A^{k} g(H)$ and $g(H) A^{k}=A^{k} g(H+k)$. But for suitably large $k, g(H)$ and $g(H+k)$ have no common zeros, so the ideal of $\mathbf{C}[H]$ generated by $g(H)$ and $g(H+k)$ is all of $\mathrm{C}[H]$. Hence the ideal of $S$ generated by $A^{k} g(H)$ and $g(H) A^{k}$ contains $A^{k}$. It follows that $I$ contains $A^{k}$ for large $k$. Similarly, $I$ contains $B^{k}$ for large $k$.

Consider the ideal $\left\langle A^{k}, B^{k}\right\rangle$ of $S$ generated by $A^{k}$ and $B^{k}$. From the expressions for $A^{k} B^{k}$ and $B^{k} A^{k}$ given in the appendix it follows that $\left\langle A^{k}, B^{k}\right\rangle$ contains

$$
\begin{gathered}
p_{k}:=(\alpha-u(H))(\alpha-u(H-1)) \cdots(\alpha-u(H-k+1)) \quad \text { and } \\
q_{k}:=(\alpha-u(H+1))(\alpha-u(H+2)) \cdots(\alpha-u(H+k)) .
\end{gathered}
$$

Let $\mathscr{Z}=\left\{\lambda_{1}, \ldots, \lambda_{n+1}\right\}$ be the zeros of $\alpha-u(H)$. Fix $m$ such that $\mathscr{Z} \subset$ $\mathscr{B}=\left\{z \in \mathbf{C}|| z \mid<\frac{1}{2} m\right\}$. Let $r$ be the greatest common divisor in $\mathbf{C}[H]$ for $p_{m}$ and $q_{m}$.

Claim. If $k \geq m$, then g.c.d. $\left(p_{k}, q_{k}\right)=r$.

Proof. Suppose that $k>m$. Write $p_{k}=p_{m} d_{k}, q_{k}=q_{m} d_{k}$. It is enough to show that g.c.d. $\left(p_{m}, d_{k}\right)=$ g.c.d. $\left(c_{k}, d_{k}\right)=$ g.c.d. $\left(q_{m}, c_{k}\right)=1$. If $g \in \mathbf{C}[H]$ write $\mathscr{Z}(g)$ for the zeros of $g$. Clearly

$$
\begin{aligned}
\mathscr{Z}\left(p_{m}\right) & =\mathscr{Z} \cup(\mathscr{Z}+1) \cup \cdots \cup(\mathscr{Z}+m-1) \\
& \subset \mathscr{B} \cup(\mathscr{B}+1) \cup \cdots \cup(\mathscr{B}+m-1), \\
\mathscr{Z}\left(q_{m}\right) & =(\mathscr{Z}-1) \cup \cdots \cup(\mathscr{Z}-m) \subset(\mathscr{B}-1) \cup \cdots \cup(\mathscr{B}-m), \\
\mathscr{Z}\left(c_{k}\right) & =(\mathscr{Z}+m) \cup \cdots \cup(\mathscr{Z}+k-1) \subset(\mathscr{B}+m) \cup \cdots \cup(\mathscr{B}+k-1), \\
\mathscr{Z}\left(d_{k}\right) & =(\mathscr{Z}-m-1) \cup \cdots \cup(\mathscr{Z}-k) \subset(\mathscr{B}-m-1) \cup \cdots \cup(\mathscr{B}-k) .
\end{aligned}
$$

It follows from the choice of $m$ that $\mathscr{Z}\left(p_{m}\right) \cap \mathscr{Z}\left(d_{k}\right)=\varnothing, \mathscr{Z}\left(c_{k}\right) \cap \mathscr{Z}\left(d_{k}\right)=\varnothing$, and $\mathscr{Z}\left(q_{m}\right) \cap \mathscr{Z}\left(c_{k}\right)=\varnothing$.

Hence if $k \geq m$, then $r \in\left\langle A^{k}, B^{k}\right\rangle$. Hence every nonzero ideal of $S$ contains $r$, and the proof is complete.

Corollary 3.8. Every nonzero ideal of $R /\langle\Omega-\alpha\rangle$ is eventually idempotent.

Corollary 3.9. The Krull dimension of $R /\langle\Omega-\alpha\rangle$ is 1 .

Proof. The proof is similar to that for the factors of $U(\operatorname{sl}(2))$. Let $I$ be the minimal nonzero ideal of $S=R /\langle\Omega-\alpha\rangle$. It is enough to show that if $0 \neq J$ is a left ideal of $S$, then $S / J$ is artinian. Using the "good behavior" of $G K$-dimension alluded to at the end of $\S 1, S / J$ has only a finite number of simple subquotients of infinite dimension. Hence (replacing $J$ if necessary) it is enough to show 
that if every proper factor of $S / J$ is finite-dimensional then $S / J$ itself is finitedimensional. Suppose that $K \supsetneqq J$. If $\operatorname{dim}_{C}(S / K)<\infty$, then $\operatorname{Ann}_{S}(S / K) \neq 0$, whence $I(S / K)=0$ and $I \subset K$; in particular, $\operatorname{dim}_{\mathbf{C}}(S / K)<\operatorname{dim}_{\mathbf{C}}(S / I)<\infty$, hence if every factor of $S / J$ is finite-dimensional, then in fact $I \subset J$, and $\operatorname{dim}_{\mathbf{C}}(S / J)<\infty$.

I expect that $R$ is of Krull dimension 2; a more complicated version of the $U(\operatorname{sl}(2))$ argument should work.

\section{A BGG CATEGORY}

The analysis of primitive ideals in enveloping algebras of semisimple Lie algebras depends on a certain category of modules discovered by Bernstein, Gelfand, and Gelfand [BGG], the category $\mathscr{O}$. There is an analog of $\mathscr{O}$ for the rings $R$. The proofs of the properties of $\mathscr{O}$ are "identical" to those given in $[\mathrm{BGG}]$.

The category $\mathscr{O}$ consists of the $R$-modules $M$ which satisfy the following conditions:

(a) $M$ is the sum of its $H$ weight spaces,

(b) for all $m \in M, \operatorname{dim}(\mathbf{C}[A] \cdot m)<\infty$,

(c) $M$ is a finitely generated $R$-module.

Thus $\mathscr{O}$ contains the Verma modules $V(\lambda)$. Clearly $\mathscr{O}$ is closed under submodules and quotient modules, so $\mathscr{O}$ contains all $L(\lambda)$.

Basic properties of modules in $\mathscr{O}$ are as follows.

Lemma 4.1. Let $M$ be an object of $\mathscr{O}$, and set $\mathscr{W}(M)=\left\{\mu \mid M_{\mu} \neq 0\right\}$. Then

(a) there exists a finite number of weights $\lambda_{0}, \ldots, \lambda_{n}$ such that $\mathscr{W}(M) \subset$ $\bigcup_{j=1}^{n}\left(\lambda_{i}-\mathbf{N}\right)$

(b) $\operatorname{dim}\left(M_{\mu}\right)<\infty$ for all $\mu$;

(c) $M$ is of finite length;

(d) if $M$ is simple then $M \cong L(\lambda)$ for some $\lambda$,

(e) if $M, N \in \mathcal{O}$, then $\operatorname{dim}_{\mathbf{C}} \operatorname{Hom}_{R}(M, N)<\infty$.

Proof. Since $M$ is finitely generated, there is a finite number of weight vectors $m_{1}, \ldots, m_{k}$ generating $M$. By hypothesis $\sum_{j} \mathbf{C}[A] m_{j}$ is a finite-dimensional $\mathrm{C}[H]$-module. Pick a basis $v_{1}, \ldots, v_{n}$ for this with $v_{i}$ of weight $\lambda_{i}$. Then (a) follows from the fact that

$$
M=\sum_{j} R m_{j}=\sum_{i} \mathbf{C}[B] \mathbf{C}[H] \mathbf{C}[A] m_{i}=\sum_{i} \mathbf{C}[B] \mathbf{C}[H] v_{i}=\sum_{i} \mathbf{C}[B] v_{i} .
$$

Since $\operatorname{dim}\left(\mathbf{C}[B] v_{i}\right)_{\mu}<\infty$ for each $i$ and each $\mu$, (b) holds.

The description of $M$ in (a) shows that $M$ is a quotient of $M\left(\lambda_{1}\right) \oplus \cdots \oplus$ $M\left(\lambda_{n}\right)$, which is of finite length.

(d) follows immediately from (a).

(e) Let $m_{1}, \ldots, m_{k}$ be weight vectors generating $M$. If $\theta \in \operatorname{Hom}_{R}(M, N)$, then $\theta$ is determined by $\theta\left(m_{1}\right), \ldots, \theta\left(m_{k}\right)$. But $\theta\left(m_{j}\right)$ is of the same weight 
as $m_{j}$. Since the weight spaces of $N$ are finite-dimensional the result follows.

If $M$ is an $R$-module, make $M^{*}$ into a left $R$-module by defining $(A \cdot \psi)(m)=\psi(B \cdot m),(B \cdot \psi)(m)=\psi(A \cdot m),(H \cdot \psi)(m)=\psi(H \cdot m)$ for all $\psi \in M^{*}, m \in M$. Thus $M \mapsto M^{*}$ is an exact contravariant functor on $R$-modules. Define $\delta M=\left\{\psi \in M^{*} \mid \operatorname{dim}(\mathbf{C}[H] \cdot \psi)<\infty\right\}$. Then $\delta$ is a (left exact) contravariant functor on the category of $R$-modules. It is easy to show that $\delta$ is exact on the category $\mathscr{O}$. But $\delta$ is not exact on the category of $R$-modules (the exactness depends on the fact that the modules in $\mathscr{O}$ are semisimple $\mathbf{C}[H]$-modules). We shall apply $\delta$ only to modules in $\mathscr{O}$.

Define an antiautomorphism of $R$ (of order 2) $r \mapsto{ }^{t} r$ by ${ }^{t} A=B,{ }^{t} B=A$, ${ }^{t} H=H$. Notice that (a) the restriction of $r \mapsto{ }^{t} r$ to $R_{0}$ is the identity, and (b) for $\psi \in M^{*}, m \in M$ we have $(r \cdot \psi)(m)=\psi\left({ }^{t} r \cdot m\right)$. Hence, if $M$ has a central character so does $\delta M$.

Lemma 4.2. Let $M \in \mathscr{O}$ :

(a) for all $\mu,(\delta M)_{\mu}=\left\{\theta \in M^{*} \mid \theta\left(M_{\nu}\right)=0\right.$ for all $\left.\nu \neq \mu\right\} \cong\left(M_{\mu}\right)^{*}$,

(b) for all $\lambda, \delta L(\lambda) \cong L(\lambda)$,

(c) $\delta M \in \mathscr{O}$,

(d) $\delta^{2} M \cong M$,

(e) the evaluation pairing $\langle\rangle:, \delta M \times M \rightarrow \mathrm{C}$ is nondegenerate, and gives an order-reversing lattice isomorphism between submodules of $M$ and of $\delta M$.

Proof. For each $\mu \in \mathscr{W}(M)$, set $N_{\mu}=\left\{\theta \in M^{*} \mid \theta\left(M_{\nu}\right)=0\right.$ for all $\left.\nu \neq \mu\right\}$. There is an injective map $N_{\mu} \rightarrow\left(M_{\mu}\right)^{*}$, so $\operatorname{dim} N_{\mu}<\infty$. It is clear that $N_{\mu}$ is a C $[H]$-submodule of $M^{*}$, hence $N_{\mu} \subset \delta M$. Furthermore, $(H-\mu) N_{\mu}=0$, so $N_{\mu} \subset(\delta M)_{\mu}$. Let $f \in(\delta M)_{\mu}$, and let $m \in M_{\nu}$ for some $\nu \neq \mu$. Then $\mu f(m)=(H \cdot f)(m)=f(H \cdot m)=f(\nu m)=\nu f(m)$, whence $f(m)=0$; therefore $f \in N_{\mu}$. It follows that $N_{\mu}=(\delta M)_{\mu}$, whence (a).

By (a), $\delta L(\lambda)$ and $L(\lambda)$ have the same weights. Hence $\delta L(\lambda)$ has a highest weight vector of weight $\lambda$. Hence there is a surjection $\delta L(\lambda) \rightarrow L(\lambda)$. Since the weights of $\delta L(\lambda)$ and $L(\lambda)$ are the same this map must be bijective. Hence (b).

By (a), $\delta M$ is a semisimple $\mathbf{C}[H]$-module, and since the weights of $\delta M$ are the same as those of $M$, if $\psi \in \delta M$, then $\operatorname{dim} \mathbf{C}[A] \cdot \psi<\infty$. Thus it remains to prove that $\delta M$ is of finite length. By (b) and (4.1d), if $M$ is simple, so too is $\delta M$. Now, since $\delta$ is an exact functor on $\mathscr{O}$, it follows by induction on the length of $M$ that the length of $\delta M$ equals that of $M$. Hence (c) holds.

There is an obvious $R$-module map $M \rightarrow \delta^{2} M$. Since $M$ and $\delta^{2} M$ have the same length, this is an isomorphism if and only if it is injective. However, if it is not injective, then there exists $0 \neq m \in M_{\mu}$, for some $\mu$, such that $\psi(m)=0$ for all $\psi \in \delta M$. But $\left(M_{\mu}\right)^{*}=(\delta M)_{\mu}$, so no such $\psi$ exists. Hence $M \cong \delta^{2} M$. 
By taking annihilators, one gets order-reversing maps between the lattices of submodules of $M$ and $\delta M$. Since $\delta M=0 \Leftrightarrow M=0$, this lattice map is bijective. Hence (e) holds.

After (4.2e), it is helpful to think of $\delta M$ as an "upside-down version of $M$."

Let $M$ be an $R$-module. For each $\alpha \in \mathbf{C}$, set $M^{\alpha}=\left\{m \in M \mid(\Omega-\alpha)^{k} m=0\right.$ for $k \gg 0\}$. Then the sum of the various $M^{\alpha}$ is direct. Let $M \in \mathscr{O}$; because $M$ is of finite length, $M=\bigoplus M^{\alpha}$. Therefore, $\mathscr{O}$ is a direct sum of subcategories, $\mathscr{O}=\bigoplus \mathscr{O}_{\alpha}$ where $\mathscr{O}_{\alpha}$ consists of those $M \in \mathscr{O}$ such that $(\Omega-\alpha)^{k} M=0$ for some $k \gg 0$. If $M=M^{\alpha}$, we say that $M$ has a generalized central character. The functor $M \mapsto \delta M$ on $\mathscr{O}$ restricts to a functor on each $\mathscr{O}_{\alpha}$. Thus each $\mathscr{O}_{\alpha}$ is self-dual.

To understand $\mathscr{O}$ one may proceed by studying each $\mathscr{O}_{\alpha}$ individually. We show that projective covers exist in $\mathscr{O}_{\alpha}$. The first requirement is a more precise version of (4.1a).

Lemma 4.3. Let $M \in \mathscr{O}_{\alpha}$. Let $L\left(\lambda_{0}\right), \ldots, L\left(\lambda_{n}\right)$ be the simple objects of $\mathscr{O}_{\alpha}$; that is, $\lambda_{0}, \ldots, \lambda_{n}$ are the zeros of $\alpha-u(\lambda+1)$. Then $\mathscr{W}(M) \subset \bigcup_{j=0}^{n}\left(\lambda_{i}-\mathbf{N}\right)$. Proof. Since $M$ has a composition series consisting of various $L\left(\lambda_{j}\right)$, and the result is true for the $L\left(\lambda_{j}\right)$, the result hoids for $M$.

Theorem 4.4. Let $M \in \mathscr{O}$. Then there exist a projective object $P \in \mathscr{O}$ and a surjective map $P \rightarrow M$.

Proof. It is enough to prove this for $M \in \mathscr{O}_{\alpha}$, so suppose $M \in \mathscr{O}_{\alpha}$. Fix $\lambda \in \mathbf{C}$. By (4.3), there exists $k \in \mathbf{N}$ (depending on $\lambda$, but not on $M$ ) such that $A^{k} M_{\lambda}=0$.

Set $I=R(H-\lambda)+R A^{k}, Q(\lambda)=R / I$, and $q=\overline{1} \in Q(\lambda)$. Clearly $Q(\lambda) \in \mathscr{O}$. Since $q$ is of weight $\lambda$, so is $\varphi(q)$ for any $\varphi \in \operatorname{Hom}_{R}(Q(\lambda), M)$. Hence the map $\operatorname{Hom}_{R}(Q(\lambda), M) \rightarrow M_{\lambda}$ defined by $\varphi \mapsto \varphi(q)$ is surjective. On the other hand, if $\varphi \neq 0$, then $\varphi(q) \neq 0$ because $q$ generates $Q(\lambda)$. Thus $\operatorname{Hom}_{R}(Q(\lambda), M) \rightarrow M_{\lambda}$ is an isomorphism. But $M \in \mathscr{O}_{\alpha}$, so $\operatorname{Hom}_{R}\left(Q(\lambda)^{\alpha}, M\right)$ $\rightarrow M_{\lambda}$ is an isomorphism.

Thus the functors $M \mapsto \operatorname{Hom}_{R}\left(Q(\lambda)^{\alpha}, M\right)$ and $M \mapsto M_{\lambda}$ on $\mathscr{O}_{\alpha}$ are isomorphic. However, $M \mapsto M_{\lambda}$ is exact on $\mathscr{O}_{\alpha}$. Hence $Q(\lambda)^{\alpha} \in \mathscr{O}_{\alpha}$ is projective (in $\mathscr{O}_{\alpha}$ ). As $M \in \mathscr{O}, M$ is finitely generated, say by elements of weights $\mu_{1}, \ldots, \mu_{k}$. Set $P=\bigoplus_{i} Q\left(\mu_{i}\right)^{\alpha}$. Thus $M$ is a homomorphic image of $P$.

Corollary 4.5. If $P \in \mathscr{O}$ is an indecomposable projective, then $P$ has a unique maximal submodule $P^{\prime}$. This gives a 1-1 correspondence between indecomposable projectives in $\mathscr{O}$ and the simple objects $L(\lambda)$ in $\mathscr{O}$. Write $P(\lambda)$ for the projective cover of $L(\lambda)$.

Proof. [BGG, Corollary 1].

Proposition 4.6. $P(\lambda)$ has a filtration by Verma modules.

Proof (see [BGG, §6]). If a direct sum of modules has a filtration by Verma modules, then so has each of the summands (the same argument as in [BGG] 
applies). Hence it is enough to show that each $Q(\lambda):=R / R(H-\lambda)+R A^{k}$ has a filtration by Verma modules. Such a filtration arises from the images of the left ideals $I_{j}:=R(H-\lambda)+R A^{j}$.

Fix $\alpha$, and let $\lambda_{0}, \ldots, \lambda_{n}$ be the zeros of $\alpha-u(\lambda+1)$. Then $P\left(\lambda_{0}\right), \ldots$, $P\left(\lambda_{n}\right)$ are the indecomposable projectives in $\mathscr{O}_{\alpha}$. Set $P=P\left(\lambda_{0}\right) \oplus \cdots \oplus P\left(\lambda_{n}\right)$ and $A=\operatorname{Hom}_{R}(P, P)$. Then $A$ is a finite-dimensional $\mathbf{C}$-algebra, and $\mathscr{O}_{\alpha}$ is equivalent to the category of finite-length $A$-modules.

The following definition is taken from [I].

A finite-dimensional C-algebra, $A$ say, is a $B G G$-algebra if the following conditions hold:

(0) there is a poset $W$ indexing the simple $A$-modules $L(w), w \in W$; write $P(w)$ for the projective cover of $L(w)$;

(1) for each $w \in W$ there is a "Verma module" $V(w)$, a finite-length $A$ module, such that $V(w)$ has simple top $L(w)$, and all other composition factors of $V(w)$ are of the form $L(y)$ with $y<w$;

(2) each $P(w)$ has a filtration by Verma modules;

(3) there is a contravariant duality functor $\delta: \operatorname{Mod} A \rightarrow \operatorname{Mod} A$ such that $\delta L(w)=L(w)$ for all $w \in W$;

(4) if $M$ is an $A$-module, having a simple top $L(w)$, and all other composition factors of the form $L(y)$ with $y$ not $>w$, then $M$ is a quotient of $V(w)$.

The category of $A$-modules is called a $B G G$-category.

Proposition 4.7. For $\alpha \in \mathbf{C}, \mathscr{O}_{\alpha}$ is a $B G G$-category for the poset $W=\{\lambda \in \mathbf{C} \mid$ $u(\lambda+1)=\alpha\}$, with the order relation $\lambda<\mu \Leftrightarrow \mu-\lambda \in \mathbf{N}$.

Proof. Only condition (4) remains to be checked. If $M \in \mathscr{O}_{\alpha}$ has simple top $L(\lambda)$, then $\operatorname{rad} M$, the radical of $M$, is the unique maximal submodule of $M$. If $M / \operatorname{Rad} M \cong L(\lambda)$, then there exists $v \in M$ of weight $\lambda$ such that the image of $v$ in $L(\lambda)$ is a highest weight vector. If all composition factors of $M$ are of the form $L(\nu)$ with $\nu<\lambda$, then every weight of $M$ is $\leq \lambda$. Hence $A \cdot v=0$. Therefore there is a map $V(\lambda) \rightarrow M$ sending the highest weight vector of $V(\lambda)$ to $v$. Therefore the composition $V(\lambda) \rightarrow M \rightarrow L(\lambda)$ is nonzero. Hence the image of $V(\lambda)$ is not contained in $\operatorname{rad} M$, whence the image is all of $M$.

The category $\mathscr{O}_{\alpha}$ may further decompose as a direct sum of subcategories. To see this, note that if either $\operatorname{Ext}^{1}(L(\lambda), L(\nu)) \neq 0$ or $\operatorname{Ext}^{1}(L(\nu), L(\lambda)) \neq 0$ then $\lambda-\nu \in \mathbf{Z}$ (this is implicit in (5.5)). Hence, $\mathscr{O}$ is the direct sum of the full subcategories $\mathscr{C}_{\sigma}$ where $\sigma \in \mathbf{C} / \mathbf{Z}$, and $\mathscr{C}_{\sigma}=\{M \in \mathscr{O} \mid \mathscr{W}(M) \subset \sigma+\mathbf{Z}\}$. Similarly, $\mathscr{O}_{\alpha}=\bigoplus_{\sigma}\left(\mathscr{O}_{\alpha} \cap \mathscr{C}_{\sigma}\right)$. Furthermore, each $\mathscr{O}_{\alpha} \cap \mathscr{C}_{\sigma}$ is a BGG-category.

This decomposition of $\mathscr{O}_{\alpha}$ corresponds to a decomposition of $W$ as a disjoint union of posets, where elements in distinct components of $W$ are incomparable. Because each Verma module $V(\lambda)$ is uniserial, each $\mathscr{O}_{\alpha}$ is a direct sum of subcategories where the index set for an indecomposable subcategory is $\{0,1, \ldots, k\} \subset \mathbf{Z}$ (for a suitable $k$ ) with its usual ordering. 
In particular, the same poset can arise from different rings $R$ and from different central characters. However, I do not know if for a fixed $W$, the BGGcategories arising from different $R$ or different central characters are equivalent or not. For example, is there a translation principle for $R$ ?

A BGG-algebra with poset $W=\{0,1, \ldots, k\}$ also arises from $U(\mathrm{sl}(k+1))$ for a suitable (singular) central character. For example, take the primitive factor of $U(\mathbf{s l}(k+1))$ which is $\mathscr{D}\left(\mathbf{P}^{k}\right)$, the ring of global differential operators on the generalized flag variety $\mathbf{P}^{k}$. The full subcategory of category $\mathscr{O}$ (for $\mathrm{sl}(k+1)$ ) consisting of those $M$ in $\mathscr{O}$ whose composition factors $L(\mu)$ are $\mathscr{D}\left(\mathbf{P}^{k}\right)$-modules is again a BGG-category. With the geometric point of view the indexing set $W$ is taken to be the set of Bruhat cells of $\mathbf{P}^{k}$ with order relation obtained through containment of closures of cells. This is the usual cellular decomposition $\mathbf{P}^{k}=\mathbf{A}^{0} \cup \mathbf{A}^{1} \cup \cdots \cup \mathbf{A}^{k}$, and the order relation is given by the inclusions of the closures of the cells $\widetilde{\mathbf{A}}^{0} \subset \overline{\mathbf{A}}^{1} \subset \cdots \subset \overline{\mathbf{A}}^{k}$. Hence the poset is $\{0,1, \ldots, k\}$ with its usual order relation.

Again, I do not know if the BGG category for $\mathscr{D}\left(\mathbf{P}^{k}\right)$ will also arise from the algebras $R$ (for suitable $f$ ).

\section{EXTENSIONS BETWEeN THE MOdULES $L(\lambda)$}

Finite-dimensional $R$-modules need not be semisimple in general; in this section we see to what extent this fails (cf. Example 2.4). The finite-dimensional $R$-modules which belong to category $\mathscr{O}$ are fairly easy to understand (as indicated at the end of $\S 4$ ), so the idea is to compare $\operatorname{Ext}_{R}^{1}$ with $\operatorname{Ext}_{\mathscr{O}}^{1}$. One of the main results $(5.5 \mathrm{a})$ in this section is that if there is a nonsplit extension between distinct finite-dimensional simple $R$-modules, then that extension occurs either at the top of a Verma module, or at the bottom of the dual of a Verma module. Thus all such extensions can be recognized from knowledge of the Verma modules. A second key result is a description of all the nonsplit extensions of a finite-dimensional simple by itself. Such an extension will not belong to category $\mathscr{O}(5.3 \mathrm{~b})$, but there is a "unique" nonsplit extension which is explicitly described in (5.11).

Example 5.1. A finite-dimensional module need not be semisimple. Set $u(x)=$ $\frac{2}{3}(x-1) x(x+1)$, so $f(x)=x(x+1)$. The submodules of $V(0)$ depend on the $j \in \mathbf{N}$ satisfying $u(1)-u(1-j)=0$. But $u(1)-u(1-j)=\frac{2}{3} j(1-j)(2-j)$, which is 0 at $j=0,1,2$. Hence there are two 1-dimensional simple modules at the top of $V(0)$. In particular, there is a nonsplit extension $0 \rightarrow L(-1) \rightarrow$ $M \rightarrow L(0) \rightarrow 0$. Note that $\operatorname{dim}_{\mathrm{C}} L(0)=\operatorname{dim}_{\mathrm{C}} L(-1)=1$.

In fact, Ann $M=\left(A, B^{2}, H(H+1)\right)$, and $R /$ Ann $M \cong$ the ring of upper triangular $2 \times 2$ matrices over $\mathbf{C}$.

Lemma 5.2. Let $M \in \mathscr{O}$. If $0 \rightarrow L(\lambda) \rightarrow M \rightarrow L(\nu) \rightarrow 0$ is a nonsplit exact sequence, then either $M$ or $\delta M$ is a highest weight module. Furthermore, $M$ has a central character. 
Proof. Suppose that neither $M$ nor $\delta M$ is a highest weight module. Note that there is an exact sequence $0 \rightarrow L(\nu) \rightarrow \delta M \rightarrow L(\lambda) \rightarrow 0$, also nonsplit.

Choose a weight vector $v \in M$ such that the image of $v$ in $L(\nu)$ is a highest weight vector. Then $v$ is of weight $\nu$, and $M=R v$. By hypothesis, $A \cdot v \neq 0$. Hence $0 \neq A v \in L(\lambda)$ is of weight $\nu+1$. Therefore $\nu+1 \leq \lambda$. The same argument applied to $\delta M$ shows that $\lambda+1 \leq \nu$.

This contradiction shows that either $M$ or $\delta M$ is a highest weight module. Since a Verma module has a central character, so does a highest weight module. Finally, if $N$ has a central character so too does $\delta N$.

Corollary 5.3. (a) If $\operatorname{dim} L(\lambda)=\operatorname{dim} L(\nu)=\infty$, then $\operatorname{Ext}_{\mathscr{O}}^{1}(L(\nu), L(\lambda))=0$.

(b) If $0 \rightarrow L(\lambda) \rightarrow M \rightarrow L(\lambda) \rightarrow 0$ is a nonsplit exact sequence, then $M$ is not in $\mathscr{O}$.

Proof. (a) This follows from (5.2) because a highest weight module has only one infinite-dimensional composition factor.

(b) This follows from (5.2) because the $\lambda$-weight space of a highest weight module is of dimension at most one.

It is not necessarily true that $\operatorname{dim} L(\lambda)=\operatorname{dim} L(\nu)=\infty \Rightarrow \operatorname{Ext}_{R}^{1}(L(\nu), L(\lambda))$ $=0$. Together, (5.2) and (5.3) say that if $\operatorname{Ext}_{\mathscr{O}}^{1}(L(\nu), L(\lambda)) \neq 0$, then at least one of the modules is finite-dimensional, and this extension occurs either at the top of $V(\nu)$ (when $\nu>\lambda)$ or at the bottom of $\delta V(\lambda)($ when $\lambda>\nu)$.

Lemma 5.4. Let $Y$ be a highest weight module of highest weight $\nu$. Let $0 \rightarrow$ $L(\lambda) \rightarrow M \rightarrow Y \rightarrow 0$ be a nonsplit exact sequence, with $\lambda \neq \nu$. Then $M \in \mathscr{O}$.

Proof. If the sequence splits the result is certainly true, so suppose not. It is clear that $M$ is of finite length and is a locally finite-dimensional $\mathbf{C}[A]$-module since $L(\lambda)$ and $Y$ have this property. Hence it suffices to prove that $M$ is a semisimple $\mathbf{C}[H]$-module. Write $\theta: M \rightarrow Y$.

Decompose $M$ into its $\mathbf{C}[H]$-primary components, i.e., $M=\bigoplus M^{\mu}$ where $M^{\mu}=\left\{m \in M \mid(H-\mu)^{k} m=0\right.$ for $\left.k \gg 0\right\}$. As $L(\lambda)$ and $Y$ are semisimple C $[H]$-modules $M^{\mu}=\left\{m \in M \mid(H-\mu)^{2} m=0\right\}$. Clearly $M^{\nu} \not \subset L(\lambda)$. Let $m \in M^{\nu} \backslash L(\lambda)$, so that $\theta(m)$ is a highest weight vector for $Y$.

If $(H-\nu) m \notin L(\lambda)$, then $\theta((H-\nu) m)$ is a highest weight vector for $Y$, so $M=L(\lambda)+R(H-\nu) m$. But then $M$ is the sum of its weight spaces, and the proof is complete.

Suppose that $(H-\nu) m \in L(\lambda)$. Since $\theta((H-\nu) m)$ is a highest weight vector, $A \cdot(H-\nu) m \in L(\lambda)$. But $(H-\nu)^{2} m=0$, so $0=A(H-\nu)^{2} m=(H-\nu-1)^{2} A m$. Since $L(\lambda)$ is a semisimple $\mathbf{C}[H]$-module, it follows that $(H-\nu-1) A m=0$. Thus $A(H-\nu) m=0$. Hence $(H-\nu) m$ is a highest weight vector for $L(\lambda)$. Thus $\lambda=\nu$. However, this contradicts our initial hypothesis.

Corollary 5.5. Suppose that $L(\lambda)$ and $L(\nu)$ are distinct finite-dimensional simple modules. Then

(a) $\operatorname{Ext}_{R}^{1}(L(\nu), L(\lambda)) \neq 0 \Leftrightarrow u(\lambda+1)=u(\nu+1)$ and either $\lambda=\nu+\operatorname{dim} L(\lambda)$, 
or $\nu=\lambda+\operatorname{dim} L(\nu)$. In this case $\operatorname{dim} \operatorname{Ext}_{R}^{1}(L(\nu), L(\lambda))=1$, and

(i) if $\nu>\lambda$ the extension is a quotient of $V(\nu)$,

(ii) if $\nu<\lambda$ the extension is a submodule of $\delta V(\lambda)$;

(b) $\operatorname{Ext}_{R}^{1}(L(\nu), L(\lambda))=\operatorname{Ext}_{\mathscr{O}}^{1}(L(\nu), L(\lambda))$;

(c) $\operatorname{Ext}_{R}^{1}(L(\nu), L(\lambda))=0 \Leftrightarrow \operatorname{Ext}_{R}^{1}(L(\lambda), L(\nu))=0$.

Proof. (a) $(\Leftrightarrow)$ If $u(\lambda+1)=u(\nu+1)$ and $\lambda=\nu+\operatorname{dim} L(\lambda)$, then a nonsplit extension exists by (2.8). If $u(\lambda+1)=u(\nu+1)$ and $\nu=\lambda+\operatorname{dim} L(\nu)$, then a nonsplit extension exists by (2.8).

(a) $(\Rightarrow)$ Let $0 \rightarrow L(\lambda) \rightarrow M \rightarrow L(\mu) \rightarrow 0$ be a nonsplit extension. By hypothesis, $\lambda \neq \nu$, so (5.3) applies with $Y=L(\nu)$. Thus $M \in \mathscr{O}$ (whence (b)), and by (5.2) either $M$ or $\delta M$ is a highest weight module. If $M$ is a highest weight module then there is a surjection $V(\nu) \rightarrow M$. If $\delta M$ is a highest weight module then there is a surjection $V(\lambda) \rightarrow \delta M$, and hence an injection $M \rightarrow \delta V(\lambda)$. Hence (i) and (ii) hold, and the other statements are consequences of this and the results in $\S 2$.

(b) Apply (5.2) and (5.4).

(c) This follows because $M$ is split $\Leftrightarrow \delta M$ is split.

Hence to understand extensions between finite-dimensional simples, the only case still to consider is $\operatorname{Ext}_{R}^{1}(L(\lambda), L(\lambda))$. Before considering $\operatorname{Ext}_{R}^{1}(L(\lambda), L(\lambda))$ some possibilities are given below as Examples 5.6a and 5.6b.

Example 5.6a. One can have $\operatorname{Ext}^{1}(L(\lambda), L(\lambda)) \neq 0$. Suppose that $u(x)=$ $\frac{1}{3} x(x-1)(2 x-1)$, thus $f(x)=x^{2}$. Set $I=R A+R B+R H$. This is a 2-sided ideal of $R$, and $L(0) \cong R / I$. Set $J=R A+R B+R H^{2}$. This is also a 2-sided ideal and $R / J \cong \mathbf{C}[t] /\left(t^{2}\right)$. The sequence $0 \rightarrow I / J \rightarrow R / J \rightarrow R / I \rightarrow 0$ is a nonsplit extension of $L(0)$ by itself. Note that $R / J$ does not have a central character: since $u(H+1)+u(H)=\frac{1}{3} H\left(4 H^{2}+2\right)$, it follows that $\Omega-\frac{1}{3} H \in I$, hence $(\Omega-\alpha)(R / J) \neq 0$ for all $\alpha \in \mathbf{C}$. In fact, whenever $f(x)=x^{n}$, there is a unique 1-dimensional $R$-module and there are always nonsplit extensions of it by itself.

Example 5.6b. In (a) the nonsplit extension $0 \rightarrow L(0) \rightarrow M \rightarrow L(0) \rightarrow 0$ was such that $M$ did not have a central character. We now show that when $f(x)=x^{3}$ one can have a nonsplit extension $0 \rightarrow L(0) \rightarrow M \rightarrow L(0) \rightarrow 0$ such that $M$ has a central character. Set $M=R / I$ where $I=\left(A, B, H^{2}\right)$. Since $f(x)=x^{3}, u(x)=\frac{1}{2} x^{2}(x-1)^{2}$. Since $u(H+1)+u(H)=H^{2}\left(H^{2}+1\right) \in I$ it follows that $\Omega \in I$, and $\Omega M=0$.

Theorem 5.7. Fix $d+1 \in \mathbf{N}$. Suppose that every zero of $u(x+1)-u(x-d)$ occurs with multiplicity 1. Suppose that $\operatorname{dim}_{\mathbf{C}} L(\lambda)=d+1$. Then $\operatorname{Ext}_{R}^{1}(L(\lambda), L(\lambda))=$ 0 .

Proof. It suffices to show that $J(\lambda)^{2}=J(\lambda)$. By (3.4) it is enough to show that $J(\lambda)_{0} \subset J(\lambda)^{2}$. Set $z=\Omega-u(\lambda+1)$ and $y=(H-\lambda)(H-\lambda+1) \cdots(H-\lambda+d)$. 
For $0<i \leq d+1$, set $a_{i}=(H-\lambda)(H-\lambda+1) \cdots(H-\lambda+d-i) A^{i}$ and $\left.b_{i}=(H-\lambda+i) \cdots(H-\lambda+d-1)(H-\lambda+d)\right) B^{i}$. Then $a_{i} b_{i} \in J(\lambda)^{2}$ and $b_{i} a_{i} \in J(\lambda)^{2}$. Using the expressions for $A^{i} B^{i}$ and $B^{i} A^{i}$ given in the appendix, it follows that

$$
\begin{aligned}
a_{i} b_{i}=\left(\frac{1}{2}\right)^{i}(H-\lambda)^{2}(H- & \lambda+1)^{2} \cdots(H-\lambda+d-i)^{2} \\
& \times(\Omega-u(H))(\Omega-u(H-1)) \cdots(\Omega-u(H-i+1))
\end{aligned}
$$

and

$$
\begin{aligned}
b_{i} a_{i}=\left(\frac{1}{2}\right)^{i}(H-\lambda+i)^{2} & (H-\lambda+i+1)^{2} \cdots(H-\lambda+d)^{2} \\
& \times(\Omega-u(H+1))(\Omega-u(H+2)) \cdots(\Omega-u(H+i)) .
\end{aligned}
$$

We must show that in $\mathbf{C}[H, \Omega],\langle y, z\rangle=\left\langle y^{2}, y z, z^{2}, a_{i} b_{i}, b_{i} a_{i}, 1 \leq i \leq\right.$ $d+1\rangle$. It is enough to work locally at the maximal ideals which lie in the support of $\langle y, z\rangle /\left\langle y^{2}, y z, z^{2}\right\rangle$. Fix $0 \leq j \leq d$, and consider the ideal $I=$ $\left\langle y^{2}, y z, z^{2}, a_{i} b_{i}, b_{i} a_{i}, \quad 1 \leq i \leq d+1\right\rangle$ locally at $\mathfrak{m}=\langle H-\lambda+j, z\rangle$. Taking the elements $a_{d-j+1} b_{d-j+1}$ and $b_{j+1} a_{j+1}$ of $I$ shows that $I_{\mathfrak{m}}$ contains

$$
(\Omega-u(H))(\Omega-u(H-1)) \cdots(\Omega-u(H-d+j))
$$

and

$$
(\Omega-u(H+1))(\Omega-u(H+2)) \cdots(\Omega-u(H+j+1)) .
$$

These elements may be rewritten as

$$
\begin{gathered}
(z+u(\lambda+1)-u(H))(z+u(\lambda+1)-u(H-1)) \\
\cdots(z+u(\lambda+1)-u(H-d+j))
\end{gathered}
$$

and

$$
\begin{gathered}
(z+u(\lambda+1)-u(H+1))(z+u(\lambda+1)-u(H+2)) \\
\cdots(z+u(\lambda+1)-u(H+j+1)) .
\end{gathered}
$$

Since $\operatorname{dim}_{\mathrm{C}} L(\lambda)=d+1$, it follows from (2.3) that $u(\lambda+1)=u(\lambda-d)$, and for all $0 \leq k<d, u(\lambda+1) \neq u(\lambda-k)$. Hence, if $0 \leq k \leq d$, then $u(\lambda+1)-u(H-k)$ is divisible by $H-\lambda+j$ if and only if $j+k=d$ or $j+k=0$. Similarly, if $0 \leq m \leq d$, then $u(\lambda+1)-u(H+m)$ is divisible by $H-\lambda+j$ if and only if $j-m=d$ or $j-m=0$.

Thus each of $u(\lambda+1)-u(H), u(\lambda+1)-u(H-1), \ldots, u(\lambda+1)-u(H-d+j+1)$ is a nonzero scalar modulo $H-\lambda+j$, and it follows that

$$
(z+u(\lambda+1)-u(H-d+j)) \in I_{\mathfrak{m}} .
$$

Similarly, each of $u(\lambda+1)-u(H+1), u(\lambda+1)-u(H+2), \ldots, u(\lambda+1)-u(H+j)$ is a nonzero scalar modulo $H-\lambda+j$, and it follows that

$$
(z+u(\lambda+1)-u(H+j+1)) \in I_{\mathrm{m}} .
$$

Therefore, $u(H+j+1)-u(H-d+j) \in I_{\mathrm{m}}$. This element of $\mathbf{C}[H]$ is divisible by $H-\lambda+j$, but only with multiplicity 1 by hypothesis. Hence 
$H-\lambda+j \in I_{\mathrm{m}}$. However, $(z+u(\lambda+1)-u(H-d+j)) \in I_{\mathrm{m}}$ and $H-\lambda+j$ divides $u(\lambda+1)-u(H-d+j)$, so $z \in I_{\mathrm{m}}$. Since $I_{\mathrm{m}}$ contains $H-\lambda+j$, which divides $y, y \in I_{\mathfrak{m}}$. Thus $I_{\mathfrak{m}}=\langle y, z\rangle_{\mathfrak{m}}$. Since $j$ was arbitrary, it follows that $I=\langle y, z\rangle$ as required.

Corollary 5.8. Fix $d+1 \in \mathbf{N}$, and suppose that $\operatorname{dim}_{\mathbf{C}} L(\lambda)=d+1<\infty$. Consider the following statements:

(a) for each $j \in \mathbf{N}$, there are $n$ simple modules of dimension $j$;

(b) each zero of $u(x+1)-u(x-d)$ occurs with multiplicity 1 ;

(c) there exist $n$ distinct highest weight modules of dimension $d+1$;

(d) $\operatorname{Ext}_{R}^{1}(L(\lambda), L(\lambda))=0$.

Then $(\mathrm{a}) \Rightarrow(\mathrm{b}) \Leftrightarrow(\mathrm{c}) \Rightarrow(\mathrm{d})$.

Proof. After (2.1) it is clear that (b) $\Leftrightarrow(c)$. That (c) $\Rightarrow(d)$ is proved in (5.7). Finally, $(\mathrm{a}) \Rightarrow(\mathrm{c})$ is obvious.

Corollary 5.9. Suppose that for each $j \in \mathbf{N}$, there are precisely $\operatorname{deg}(f)$ simple modules of dimension $j$. Then every finite-dimensional $R$-module is semisimple. Proof. Let $L(\lambda)$ be finite-dimensional. By (5.8), $\operatorname{Ext}_{R}^{1}(L(\lambda), L(\lambda))=0$. On the other hand, if $\lambda \neq \nu$, and $L(\nu)$ is finite-dimensional, then $\operatorname{Ext}_{R}^{1}(L(\nu), L(\lambda)) \neq$ 0 implies by $(5.5 \mathrm{a})$ that either length $V(\lambda) \geq 3$ or length $V(\nu) \geq 3$. But this is impossible by $(2.3 \mathrm{c})$.

In $\S 2$, I expressed the expectation that the equivalent conditions of (2.5) would always hold. If that expectation is fulfilled, then a modification of (5.8) and (5.9) would say that "most" finite-dimensional $R$-modules are semisimple.

Example 5.10. Set $u(x)=x^{n+1}$, with $n \geq 2$. It is instructive to compare the properties of $R$ and $U(\operatorname{sl}(2))$. We will show that (i) for each $k \in \mathbf{N}$ there are precisely $n$ simple modules of dimension $k$, and (ii) every finite-dimensional $R$-module is a direct sum of simple modules.

Set $Z=\left\{\omega \in \mathbf{C} \mid \omega^{n+1}=1, \omega \neq 1\right\}$, and $\mathscr{Z}=\left\{(\omega-1)^{-1} \mid \omega \cdot \in Z\right\}=$ $\left\{z_{1}, \ldots, z_{n}\right\}$.

(a) Claim. $V(\lambda)$ has a quotient module of dimension $j \in \mathbf{N} \Leftrightarrow \lambda+1=$ $j(z+1)$ for some $z \in \mathscr{Z}$. The nonsimple $V(\lambda)$ are precisely $\{V(\lambda) \mid \lambda+1 \in$ $(z+1) \mathbf{N}$ for some $z \in \mathscr{Z}\}$. These $\lambda$ lie on $n$ (real) lines (passing through the point -1 ) in the complex plane $\mathbf{C}$, which do not intersect i.e. they are distinct. Hence there are precisely $n$ distinct highest weight modules of dimension $j$, by $(\mathrm{a}) \Rightarrow$ (c) of $(5.8)$.

Proof. Set $p(z)=(z+1)^{n+1}-z^{n+1} \in \mathbf{C}[z]$. Then $u(\lambda+1)-u(\lambda-j+1)=$ $j^{n+1} p\left(j^{-1}(\lambda+1)-1\right)$. Hence $V(\lambda)$ has a submodule isomorphic to $V(\lambda-j)$ (and a quotient module of dimension $j$ ) if and only if $p\left(j^{-1}(\lambda+1)-1\right)=0$. But $p(z)=0 \Leftrightarrow z=(\omega-1)^{-1}$ for some $\omega \in Z \Leftrightarrow z \in \mathscr{Z}$. Hence, $V(\lambda)$ has a $j$-dimensional quotient $\Leftrightarrow j^{-1}(\lambda+1)-1 \in \mathscr{Z} \Leftrightarrow \lambda=j(z+1)-1$ for some $z \in \mathscr{Z}$. 
To see that the lines $(z+1) \mathbf{R}-1(z \in \mathscr{Z})$ distinct, it is enough to show that arguments of the complex numbers $z_{1}+1, \ldots, z_{n}+1$ are distinct. If $\omega=\cos \theta+i \sin \theta(\theta=2 \pi m /(n+1), 1 \leq m \leq n)$ then $z_{m}+1=(\omega-1)^{-1}+$ $1=\frac{1}{2}\left(1-i(\sin \theta)(1-\cos \theta)^{-1}\right)$. It is clear that these have distinct arguments. Therefore the lines $\left(z_{k}+1\right) \mathbf{R}$ distinct.

(b) Claim. Each $V(\lambda)$ is of length $\leq 2$.

Proof. Fix $\lambda$. Suppose that length $V(\lambda)>2$. Then there exist $j_{1} \neq j_{2}$ and two distinct $z_{1}, z_{2} \in \mathscr{Z}$ (after renumbering the roots) with $\lambda+1=j_{1} z_{1}=j_{2} z_{2}$. Hence the lines $z_{1} \mathbf{R}$ and $z_{2} \mathbf{R}$ are not distinct, contradicting (a). So, for all $\lambda \in \mathbf{C}$, length $V(\lambda) \leq 2$.

(c) Claim. (i) For each $j \in \mathbf{N}$, there are precisely $n$ simple modules of dimension $j$. (ii) Every finite-dimensional $R$-module is semisimple.

Proof. (i) By (a) there are precisely $n$ distinct $V(\lambda)$ having a $j$-dimensional quotient. By (b) such a quotient must be simple.

(ii) Apply (5.9).

Consider the case $u(x)=x^{8}$; that is, $f(x)=(x+1)^{8}-x^{8}$. The points in the set $\left\{\lambda \in \mathbf{C} \mid \operatorname{dim}_{\mathbf{C}} L(\lambda)<\infty\right\}$ are plotted in Figure 1. These are the points $\left(z_{m}+1\right) \mathbf{N}-1$ which lie on the lines $\left(z_{m}+1\right) \mathbf{R}-1$, where $z_{m}+1=$ $\frac{1}{2}\left(1-\sin \left(\theta_{m}\right)\left(1-\cos \left(\theta_{m}\right)\right)^{-1}\right)$ and $\theta_{m}=2 \pi m / 8,1 \leq m \leq 7$. Beside each point $\lambda$ is written $\operatorname{dim}_{\mathrm{C}} L(\lambda)$.

Proposition 5.11. Fix $d+1 \in \mathbf{N}$. Suppose $x=\lambda$ is a zero of $u(x+1)-u(x-d)$ of multiplicity $>1$. Suppose that $\operatorname{dim}_{\mathbf{C}} L(\lambda)=d+1$. Then $\operatorname{Ext}_{R}^{1}(L(\lambda), L(\lambda))=\mathbf{C}$, and the "unique" nonsplit extension of $L(\lambda)$ by itself is $R / I$ where $I=R A+$ $R B^{d+1}+R(H-\lambda)^{2}$.

Proof. Now $I \subset I^{\prime}:=R A+R B^{d+1}+R(H-\lambda)$, and $I^{\prime}$ is the annihilator of the highest weight vector in $L(\lambda)$, whence $R / I^{\prime} \cong L(\lambda)$. Similarly, $I^{\prime} / I$ is generated by the image of $(H-\lambda)$ which is annihilated by $I^{\prime}$, so there is a surjective map $L(\lambda) \rightarrow I^{\prime} / I$. The next step is to show that $I \neq I^{\prime}$.

It suffices to show that $(H-\lambda) \notin I$. Consider

$$
I \cap R_{0}=R_{0} B A+R_{0} A^{d+1} B^{d+1}+R_{0}(H-\lambda)^{2} .
$$

Recall that $R_{0}=\mathbf{C}[H, \Omega], \quad B A=\frac{1}{2}(\Omega-u(H+1))$, and $A^{d+1} B^{d+1}$ $=\left(\frac{1}{2}\right)^{d+1}(\Omega-u(H))(\Omega-u(H-1)) \cdots(\Omega-u(H-d))$. Hence, $I \cap R_{0}$ is generated by $\Omega-u(H+1),(H-\lambda)^{2}$, and $(\Omega-u(H))(\Omega-u(H-1)) \cdots(\Omega-u(H-d))$ as an ideal of $R_{0}$. By hypothesis, $u(H+1)-u(H-d) \in\left\langle(H-\lambda)^{2}\right\rangle$. Hence $\Omega-u(H-d) \in\left\langle\Omega-u(H+1),(H-\lambda)^{2}\right\rangle$. Thus $I \cap R_{0}=\left\langle\Omega-u(H+1),(H-\lambda)^{2}\right\rangle$. Since $\Omega$ and $H$ are algebraically independent, it follows that $(H-\lambda) \notin I \cap R_{0}$.

Thus $I \neq I^{\prime}$, and $I^{\prime} / I \cong L(\lambda)$. Hence there is a short exact sequence $0 \rightarrow L(\lambda) \rightarrow R / I \rightarrow L(\lambda) \rightarrow 0$. It is clear that this is nonsplit, since if it 


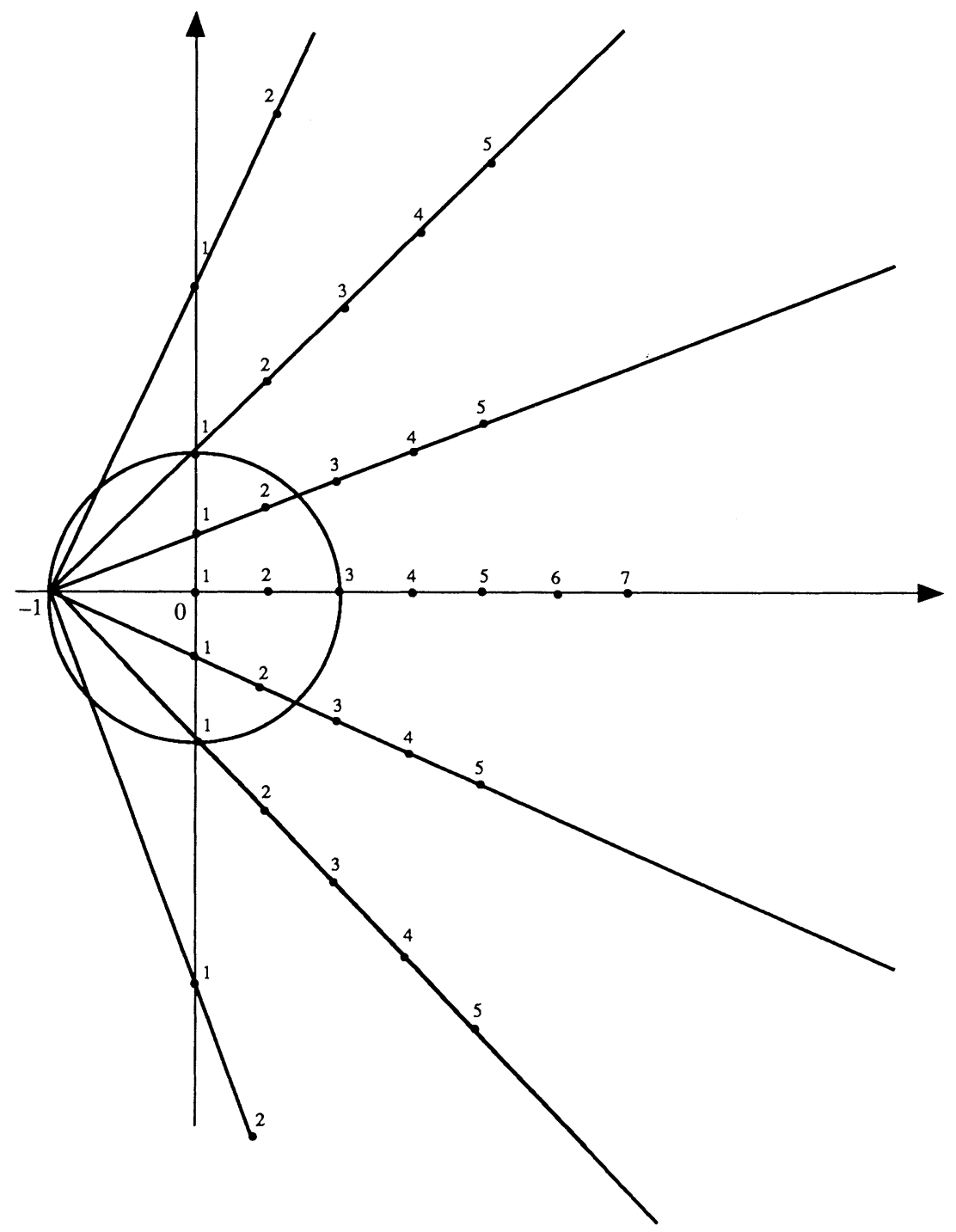

Figure 1. The points $\lambda \in \mathbf{C}$ such that $\operatorname{dim}_{\mathbf{C}} L(\lambda)<\infty$ when $f(n)=(x+1)^{8}-x^{8}$

were $R / I$ would be the sum of its weight spaces; this is not the case, since $(H-\lambda)^{2} \in I$ but $(H-\lambda) \notin I$.

It remains to show that $R / I$ is the only nonsplit extension of $L(\lambda)$ by $L(\lambda)$. Suppose that $0 \rightarrow L(\lambda) \rightarrow M \rightarrow L(\lambda) \rightarrow 0$ is nonsplit. We will make use of the $\mathrm{C}[H]$-primary decomposition of $M$. Choose $e \in M$ such that $\bar{e} \in M / L(\lambda) \cong$ $L(\lambda)$ is of weight $\lambda$. Thus $(H-\lambda) e \in L(\lambda)$. By (5.3), $M$ is not in $\mathscr{O}$, so $M$ is not the sum of its weight spaces. Therefore $M$ is not generated by a weight vector. Hence, $(H-\lambda) e \neq 0$. Therefore there exists $e \in M \backslash L(\lambda)$ such that $(H-\lambda)^{2} e=0$, and $(H-\lambda) e \neq 0$. Now $0=A(H-\lambda)^{2} e=(H-\lambda-1)^{2} A e$, but 
the $\langle H-\lambda-1\rangle$-primary component of $L(\lambda)$, and hence of $M$, is zero. Thus $A e=0$. A similar argument, using the fact that there is no $\langle H-\lambda+d+1\rangle$ primary component, shows that $B^{d+1} e=0$. Since $M$ is nonsplit $M=R e \cong$ $R / \operatorname{Ann}(e)$. But we have just shown that $I \subset \operatorname{Ann}(e)$, and the result follows from the first part of the proof.

\section{The CASE degree $(f)=2$}

After (1.1), if $\operatorname{deg}(f) \leq 1$, then $R$ is "known." Hence the first new case is $\operatorname{deg}(f)=2$, or equivalently $\operatorname{deg}(u)=3$. In this section we study this case in detail.

It is necessary only to study $R$ up to isomorphism, so, as the next lemma indicates, we can assume that $f$ is of a special form. Fix $c \in \mathbf{C}$, and set $u(x)=$ $x^{3}+c x$. Then $f(x)=\frac{1}{2}(\Delta u)(x)=\frac{1}{2}\left(3 x^{2}+3 x+1+c\right)$. Any $f(x)$ of degree 2 can be brought into this form by a change of the form $f(x) \mapsto \alpha f(x+\beta)$; the point of (6.1) below is that such a change to $f$ does not change the isomorphism class of $R$. Hence, if $\operatorname{deg}(f)=2$, then there exists $c \in \mathbf{C}$ such that the $R$ determined by $f$ is isomorphic to the $R$ determined by $u(x)=x^{3}+c x$. The case $u(x)=x^{3}$ is part of (5.10). So throughout $\S 6$, take $u(x)=x^{3}+c x$.

Lemma 6.1. For $i=1,2$, let $f_{i} \in \mathbf{C}[x]$. Set $R_{i}=\mathbf{C}\left[A_{i}, B_{i}, H_{i}\right]$ subject to the usual defining relations with $\left[A_{i}, B_{i}\right]=f_{i}\left(H_{i}\right)$.

(a) If $\alpha f_{1}=f_{2}$ for $0 \neq \alpha \in \mathbf{C}$, then $R_{1} \cong R_{2}$ via $A_{1} \rightarrow \alpha A_{2}, B_{1} \rightarrow B_{2}$, $H_{1} \rightarrow H_{2}$.

(b) If $f_{1}(x+\alpha)=f_{2}(x)$ for $0 \neq \alpha \in \mathbf{C}$, then $R_{1} \cong R_{2}$ via $A_{1} \rightarrow A_{2}$, $B_{1} \rightarrow B_{2}, H_{1} \rightarrow H_{2}+\alpha$.

Proof. Easy.

Remarks. (1) Note that $\alpha f_{1}=f_{2}$ is equivalent to $\alpha u_{1}=u_{2}$, and $f_{1}(x+\alpha)=$ $f_{2}(x)$ is equivalent to $u_{1}(x+\alpha)=u_{2}(x)$.

(2) I believe it will be very difficult to understand precisely when $R_{1} \cong R_{2}$. Nevertheless, if the conjecture in $\S 2$ is correct then $R$ does determine $\operatorname{deg}(f)$ because the number of $j$-dimensional simple modules is precisely $n$ for $j \gg 0$

Since $u(x)=x^{3}+c x, u(x+1)-u(x+1-y)=y\left\{3(x+1)^{2}-3(x+1) y+y^{2}+c\right\}$. For $j \in \mathbf{N}$, the set of $V(\lambda)$ with a $j$-dimensional quotient is precisely

$$
\left\{V(\lambda) \mid 3(\lambda+1)^{2}-3(\lambda+1) j+j^{2}+c=0\right\} .
$$

Since $\operatorname{deg}(u)=3$, there are at most three Verma modules with a given central character, a Verma module has length at most $3(2.2 \mathrm{a})$, and for each $j \in \mathbf{N}$ there are at most two simple modules of dimension $j$.

(a) Claim. $R$ has a Verma module of length 3 if and only if there exist distinct $i, k \in \mathbf{N}$ such that $c=-\frac{1}{3}\left(i^{2}-i k+k^{2}\right)$. Suppose that $(i, k) \in \mathbf{N}^{2}$ is a solution to $c=-\frac{1}{3}\left(i^{2}-i k+k^{2}\right)$, with $i>k$. Then $V\left(\frac{1}{3}(i+k)-1\right)$ has composition factors $L\left(\frac{1}{3}(i+k)-1\right)$, which is of dimension $k ; L\left(\frac{1}{3}(i-2 k)-1\right)$, 
which is of dimension $i-k$; and $L\left(\frac{1}{3}(k-2 i)-1\right)$, which is infinite-dimensional. Furthermore, one has $c=-\frac{1}{3}\left(i^{2}-i(i-k)+(i-k)^{2}\right)$, so $V\left(\frac{1}{3}(2 i-k)-1\right)$ is also of length 3. This has composition factors $L\left(\frac{1}{3}(2 i-k)-1\right)$, which is of dimension $i-k ; L\left(\frac{1}{3}(2 k-i)-1\right)$, which is of dimension $k$; and $L\left(\frac{1}{3}(-i-k)-1\right)$, which is infinite-dimensional.

Proof. Let $\lambda \in \mathbf{C}$. From the above, $V(\lambda)$ is of length $3 \Leftrightarrow$ there exist quotients of $V(\lambda)$ of dimensions $k$ and $i$ say, with $k>i \Leftrightarrow$ there are distinct $i, k \in \mathbf{N}$ such that $3(\lambda+1)^{2}-3(\lambda+1) i+i^{2}+c=3(\lambda+1)^{2}-3(\lambda+1) k+k^{2}+c=0$. Now $(j-i)(j-k)=3(\lambda+1)^{2}-3(\lambda+1) j+j^{2}+c \Leftrightarrow i+k=3(\lambda+1)$, and $i k=3(\lambda+1)^{2}+c \Leftrightarrow c=-\frac{1}{3}\left(i^{2}-i k+k^{2}\right)$ and $\lambda+1=\frac{1}{3}(i+k)$.

Finding the composition series is obvious.

There are at most a finite number of $V(\lambda)$ of length 3 , because there is only a finite number of ways to write $c$ as $c=-\frac{1}{3}\left(i^{2}-i k+k^{2}\right)$ with $i, k \in \mathbf{N}$.

(b) Fix $j \in \mathbf{N}$. Claim. There are two distinct highest weight modules of dimension $j \Leftrightarrow c \neq-\frac{1}{4} j^{2}$.

Proof. $V(\lambda)$ has a $j$-dimensional quotient $\Leftrightarrow x=\lambda$ is a zero of $3(x+1)^{2}-$ $3(x+1) j+j^{2}+c$. Hence there are two distinct $j$-dimensional highest weight modules $\Leftrightarrow$ the discriminant of this polynomial in $x$ does not vanish $\Leftrightarrow c \neq$ $-\frac{1}{4} j^{2}$.

(c) Claim. Suppose that $c=-\frac{1}{4} j^{2}$. Then there is either (when $j$ is odd) a unique $j$-dimensional simple module, namely, $L\left(\frac{1}{2} j-1\right)$, or (when $j$ is even) no $j$-dimensional simple module.

Proof. $V(\lambda)$ has a $j$-dimensional quotient $\Leftrightarrow 3(\lambda+1)^{2}-3(\lambda+1) j+j^{2}+c=0 \Leftrightarrow$ $3(\lambda+1)^{2}-3(\lambda+1) j+j^{2}-\frac{1}{4} j^{2}=0 \Leftrightarrow \lambda=\frac{1}{2} j-1$. The module $V\left(\frac{1}{2} j-1\right)$ has a quotient of dimension $i \Leftrightarrow 3\left(\frac{1}{2} j\right)^{2}-3\left(\frac{1}{2} j\right) i+i^{2}-\frac{1}{4} j^{2}=0 \Leftrightarrow(j-i)(j-2 i)=0$. Hence, if $j$ is odd, $V\left(\frac{1}{2} j-1\right)$ has only one finite-dimensional quotient, namely $L\left(\frac{1}{2} j-1\right)$, which is of dimension $j$. However, if $j$ is even, then $V\left(\frac{1}{2} j-1\right)$ is of length 3 (see case 4 below); the composition factors of $V\left(\frac{1}{2} j-1\right)$ are $L\left(\frac{1}{2} j-1\right)$, which is of dimension $\frac{1}{2} j, L(-1)$, which is of dimension $\frac{1}{2} j$, and $L\left(-\frac{1}{2} j-1\right)$, which is infinite-dimensional.

It is now possible to analyze all the possibilities. Define

$$
\Gamma=\left\{-\frac{1}{3}\left(j_{1}^{2}-j_{1} j_{2}+j_{2}^{2}\right) \mid j_{1}, j_{2} \in \mathbf{N}\right\} \quad \text { and } \Lambda=\left\{-\frac{1}{4} j^{2} \mid j \in \mathbf{N}\right\} .
$$

(1) Suppose that $c \notin \Lambda$ and $c \notin \Gamma$ (this is the generic case and, in particular, will occur whenever $c \geq 0$ ). There are precisely two simple modules of dimension $j$ for every $j \in \mathbf{N}$, and every finite-dimensional $R$-module is semisimple. This follows from (b) and (5.9).

(2) Suppose that $c \notin \Lambda$ and $c \in \Gamma$ (e.g., $c=-7 / 3$ ). By (b), there are precisely two highest weight modules of dimension $j$ for every $j \in \mathbf{N}$. However, some of these will not be simple. If $c=-\frac{1}{3}\left(k^{2}-i k+i^{2}\right)$ with $i>k \in \mathbf{N}$, then 
$V\left(\frac{1}{3}(i+k)-1\right)$ and $V\left(\frac{1}{3}(2 i-k)-1\right)$ are both of length 3 and have $i$-dimensional quotients which are not simple. Note that $i \neq 2 k$, else $c \in \Lambda$. There are no simple modules of dimension $i$, because the two distinct $i$-dimensional highest weight modules are the length 2 quotients of $V\left(\frac{1}{3}(i+k)-1\right)$ and $V\left(\frac{1}{3}(2 i-k)-1\right)$. After $(5.5 \mathrm{a})$ the nonsplit finite-dimensional modules of length 2 arise from the Verma modules of length 3. By (5.7), $\operatorname{Ext}_{R}^{1}(L(\lambda), L(\lambda))=0$ for all finite-dimensional $L(\lambda)$.

(3) Suppose that $m \in \mathbf{N}$ and that $c=-\frac{1}{4}(2 m+1)^{2} \in \Lambda$. A simple calculation shows that $c \notin \Gamma$. There is exactly one simple module of dimension $2 m+1$, namely $L(m)$. For every other $j \in \mathbf{N}$, there are exactly two simple modules of dimension $j$. There is a unique nonsplit finite-dimensional module of length two, and it arises as $0 \rightarrow L(m) \rightarrow M \rightarrow L(m) \rightarrow 0$.

(4) Suppose that $m \in \mathbf{N}$ and that $c=-\frac{1}{4}(2 m)^{2} \in \Lambda$. A simple calculation shows that a solution to $c=-\frac{1}{3}\left(k^{2}-i k+i^{2}\right)$ with $i, k \in \mathbf{N}$ is given by $i=2 m, k=m$ (there may be other solutions). There is no simple module of dimension $2 m$. For every other $j \in \mathbf{N}$ there is at least one simple module of dimension $j$; there will be two simple modules of dimension $j$ unless there is a solution to $c=-\frac{1}{3}\left(k^{2}-j k+j^{2}\right)$ with $j>k \in \mathbf{N}$. The two $m$-dimensional simple modules are $L(m-1)$ and $L(-1)$, and there is a nonsplit extension $0 \rightarrow L(-1) \rightarrow M \rightarrow L(m-1) \rightarrow 0$ which occurs at the top of $V(m-1)$. This extension is in some sense "trying to be the missing $2 m$-dimensional simple module."

\section{APPENDIX}

Useful identities:

$$
\begin{aligned}
& f(x)+f(x-1)+\cdots+f(x-j+1)=\frac{1}{2}(u(x+1)-u(x-j+1)) \quad \text { for } j \in \mathbf{N} . \\
& g(H) A=A g(H+1) \\
& g(H) B=B g(H-1) \\
& {[g(H), A]=A(g(H+1)-g(H))=A \Delta(g(H)),} \\
& {[g(H), B]=B(g(H-1)-g(H))=-B \Delta(g(H-1)),} \\
& \left.\left[\begin{array}{c}
H \\
n
\end{array}\right), A\right]=A\left(\begin{array}{c}
H \\
n-1
\end{array}\right) \\
& A B-B A=\frac{1}{2}(u(H+1)-u(H)) \\
& A B+B A=\Omega-\frac{1}{2}(u(H+1)+u(H)) \\
& A B=\frac{1}{2}(\Omega-u(H)) \\
& B A=\frac{1}{2}(\Omega-u(H+1)) \\
& A^{k} B^{k}=\frac{1}{2} A^{k-1}(\Omega-u(H)) B^{k-1} \\
& \quad=\frac{1}{2} A^{k-1} B^{k-1}(\Omega-u(H-k+1)) \\
& \quad=\left(\frac{1}{2}\right)^{k}(\Omega-u(H))(\Omega-u(H-1)) \cdots(\Omega-u(H-k+1))
\end{aligned}
$$




$$
\begin{aligned}
& \begin{aligned}
B^{k} A^{k} & =\frac{1}{2} B^{k-1}(\Omega-u(H+1)) A^{k-1} \\
& =\frac{1}{2} B^{k-1} A^{k-1}(\Omega-u(H+k)) \\
& =\left(\frac{1}{2}\right)^{k}(\Omega-u(H+1))(\Omega-u(H+2)) \cdots(\Omega-u(H+k)),
\end{aligned} \\
& {\left[g(H), A^{k}\right]=A^{k}(g(H+k)-g(H)),} \\
& {\left[g(H), B^{k}\right]=B^{k}(g(H-k)-g(H)) .}
\end{aligned}
$$

\section{REFERENCES}

[AHV] J. Alev, T. J. Hodges, and J. D. Velez, Fixed rings of the Weyl algebra $A_{1}(\mathbf{C})$, J. Algebra (to appear).

[AS] M. Artin and W. Schelter, Graded algebras of global dimension 3, Adv. in Math. 66 (1987), 171-216.

[BS] A. Bell and S. P. Smith, Some 3-dimensional skew polynomial rings, in preparation.

[B] G. M. Bergman, The Diamond lemma for ring theory, Adv. in Math. 29 (1978), 178-218.

[BGG] J. Bernstein, I. M. Gelfand, and S. I. Gelfand, A category of $\mathfrak{g}$-modules, Functional Anal. Appl. 10 (1976), 87-92.

[C] P. M. Cohn, Algebra, Vol. 2, Wiley, New York, 1977.

[D] J. Dixmier, Enveloping algebras, North-Holland, Amsterdam, 1977.

[H1] T. J. Hodges, Letter, November 1987.

[H2] _ Letter, August 1988.

[HS] T. J. Hodges and S. P. Smith, Sheaves of non-commutative algebras and the BeilinsonBernstein equivalence of categories, Proc. Amer. Math. Soc. 93 (1985), 379-386.

[I] R. S. Irving, $B G G$ algebras and the $B G G$ reciprocity principle, J. Algebra (to appear).

[J] A. Joseph, Rings which are modules in the Bernstein-Gelfand-Gelfand $\mathscr{O}$-category, J. Algebra 113 (1988), 110-126.

[KL] G. Krause and T. H. Lenagan, Gelfand-Kirillov dimension and growth of algebras, Pitman, London, 1984.

[L.] S. Lang, Diophantine geometry, Interscience, New York, 1962.

[LSS] T. Levasseur, S. P. Smith, and J. T. Stafford, The minimal nilpotent orbit, the Joseph ideal and differential operators, J. Algebra 116 (1988), 480-501.

[LS] T. Levasseur and J. T. Stafford, Differential operators on classical rings of invariants, Mem. Amer. Math. Soc. No. 412 (1989).

[MR] J. C. McConnell and J. C. Robson, Gelfand-Kirillov dimension, Hilbert-Samuel polynomials, and rings of differential operators, preprint, Univ. of Leeds, 1987.

[MS] J. C. McConnell and J. T. Stafford, Gelfand-Kirillov dimension, and associated graded modules, J. Algebra 125 (1989), 197-214.

[SW] L. Small and R. Warfield, Prime affine algebras of Gelfand-Kirillov dimension 1, J. Algebra 91 (1984), 386-389.

[Sm] S. P. Smith, Overrings of primitive factor rings of $U(\mathrm{sl}(2, \mathbf{C}))$, J. Pure Appl. Algebra (to appear).

[St] J. T. Stafford, Homological properties of $U(\mathrm{sl}(2))$, Proc. Cambridge Philos. Soc. 91 (1982), 29-37.

Department of Mathematics, University of Washington, Seattle, Washington 98195 